\title{
GAS DISTRIBUTION, KINEMATICS, AND EXCITATION STRUCTURE IN THE DISKS AROUND THE CLASSICAL Be STARS $\beta$ CANIS MINORIS AND $\zeta$ TAURI*
}

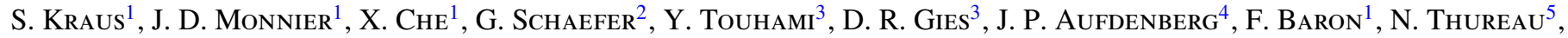 \\ T. A. Ten BrummelaAR ${ }^{2}$, H. A. McAlister ${ }^{2}$, N. H. Turner ${ }^{2}$, J. Sturmann ${ }^{2}$, And L. Sturmann ${ }^{2}$ \\ ${ }^{1}$ Department of Astronomy, University of Michigan, 918 Dennison Building, Ann Arbor, MI 48109-1090, USA; stefankr@umich.edu \\ ${ }^{2}$ The CHARA Array, Georgia State University, P.O. Box 3965, Atlanta, GA 30302-3965, USA \\ ${ }^{3}$ Center for High Angular Resolution Astronomy and Department of Physics and Astronomy, Georgia State University, P.O. Box 4106, \\ Atlanta, GA 30302-4106, USA \\ ${ }^{4}$ Department of Physical Sciences, Embry-Riddle Aeronautical University, 600 S. Clyde Morris Blvd., Daytona Beach FL 32114, USA \\ ${ }^{5}$ Department of Physics and Astronomy, University of St. Andrews, UK \\ Received 2011 May 23; accepted 2011 September 11; published 2011 December 8
}

\begin{abstract}
Using CHARA and VLTI near-infrared spectro-interferometry with hectometric baseline lengths (up to $330 \mathrm{~m}$ ) and with high spectral resolution (up to $\lambda / \Delta \lambda=12,000$ ), we studied the gas distribution and kinematics around two classical Be stars. The combination of high spatial and spectral resolution achieved allows us to constrain the gas velocity field on scales of a few stellar radii and to obtain, for the first time in optical interferometry, a dynamical mass estimate using the position-velocity analysis technique known from radio astronomy. For our first target star, $\beta$ Canis Minoris, we model the $H+K$-band continuum and $\mathrm{Br} \gamma$-line geometry with a near-critical rotating stellar photosphere and a geometrically thin equatorial disk. Testing different disk rotation laws, we find that the disk is in Keplerian rotation $\left(v(r) \propto r^{-0.5 \pm 0.1}\right)$ and derive the disk position angle $\left(140^{\circ} \pm 1.7\right)$, inclination $\left(38.5 \pm 1^{\circ}\right)$, and the mass of the central star $\left(3.5 \pm 0.2 M_{\odot}\right)$. As a second target star, we observed the prototypical Be star $\zeta$ Tauri and spatially resolved the $\mathrm{Br} \gamma$ emission as well as nine transitions from the hydrogen Pfund series (Pf 14-22). Comparing the spatial origin of the different line transitions, we find that the Brackett $(\mathrm{Br} \gamma)$, Pfund (Pf 14-17), and Balmer ( $\mathrm{H} \alpha)$ lines originate from different stellocentric radii $\left(R_{\mathrm{cont}}<R_{\mathrm{Pf}}<R_{\mathrm{Br} \gamma} \sim R_{\mathrm{H} \alpha}\right)$, which we can reproduce with an LTE line radiative transfer computation. Discussing different disk-formation scenarios, we conclude that our constraints are inconsistent with wind compression models predicting a strong outflowing velocity component, but support viscous decretion disk models, where the Keplerian-rotating disk is replenished with material from the near-critical rotating star.
\end{abstract}

Key words: circumstellar matter - stars: emission-line, Be - stars: fundamental parameters - stars: individual (Beta CMi, Zeta Tau) - techniques: interferometric

Online-only material: color figures

\section{INTRODUCTION}

Classical Be stars are main-sequence (or near main-sequence) B-type stars associated with hydrogen line emission, indicating the presence of ionized circumstellar gas, which is believed to be arranged in an equatorial disk-like structure. Optical/infrared spectroscopic observations have revealed non-radial pulsations (e.g., Rivinius et al. 1998), which might provide a way to feed material from the photosphere to the inner disk. Polarimetric studies (e.g., Draper et al. 2011) can constrain the disk density structure, while spectro-astrometry (e.g., Oudmaijer et al. 2011) can provide information about the disk kinematics in the spatially unresolved regime. Further unique insights into the structure and physics of these disks can also be obtained with interferometry at visual and infrared wavelengths, allowing one to unravel the inner disk structure on scales of a few stellar radii directly. For instance, interferometric studies have allowed associating quasi-cyclic variations in the ratio between the blue- and red-shifted wing of the $\mathrm{H} \alpha$-line emission $(V / R$ variability) with global oscillations in the circumstellar disk, likely in the form of a one-armed spiral density pattern (e.g., Vakili et al. 1998; Stefl et al. 2009; Carciofi et al. 2009; Schaefer et al. 2010). Besides

\footnotetext{
* Based on observations made with ESO telescopes at the Paranal Observatory under programme IDs 084.C-0848(A) and 085.C-0911(A) and with the CHARA Array.
}

studies on the disk continuum geometry, interferometric observations in spectral lines have provided the first direct constraints on the gas kinematics, in particular for the hydrogen spectral lines of the Balmer (e.g., Quirrenbach et al. 1994; Vakili et al. 1998; Tycner et al. 2005; Delaa et al. 2011), Brackett (Meilland et al. 2007, 2011), and Pfund series (Pott et al. 2010). These studies have provided growing evidence that the disks around classical Be stars exhibit a near-Keplerian rotation profile, which might allow to effectively rule out several disk-formation scenarios (e.g., see review by Carciofi 2010). However, most earlier studies using spectro-interferometry were limited in terms of spectral resolution or baseline position angle (PA) coverage, leaving significant uncertainties about the detailed gas velocity field and the evidence to distinguish between a purely rotational versus an expanding velocity component in the disk. Obtaining such evidence is essential in order to decide between different scenarios which have been proposed to explain the disk-formation mechanism, including radiatively driven winds, ram pressure or magnetically induced wind compression, and viscous decretion (Porter \& Rivinius 2003). Furthermore, recently there has been a controversy about the appearance of a phase inversion in spectro-interferometric observations of several classical Be stars, which triggered speculations about secondary dynamical effects or the need for an additional kinematical component beyond the canonical star+disk paradigm (Stefl et al. 2011). 


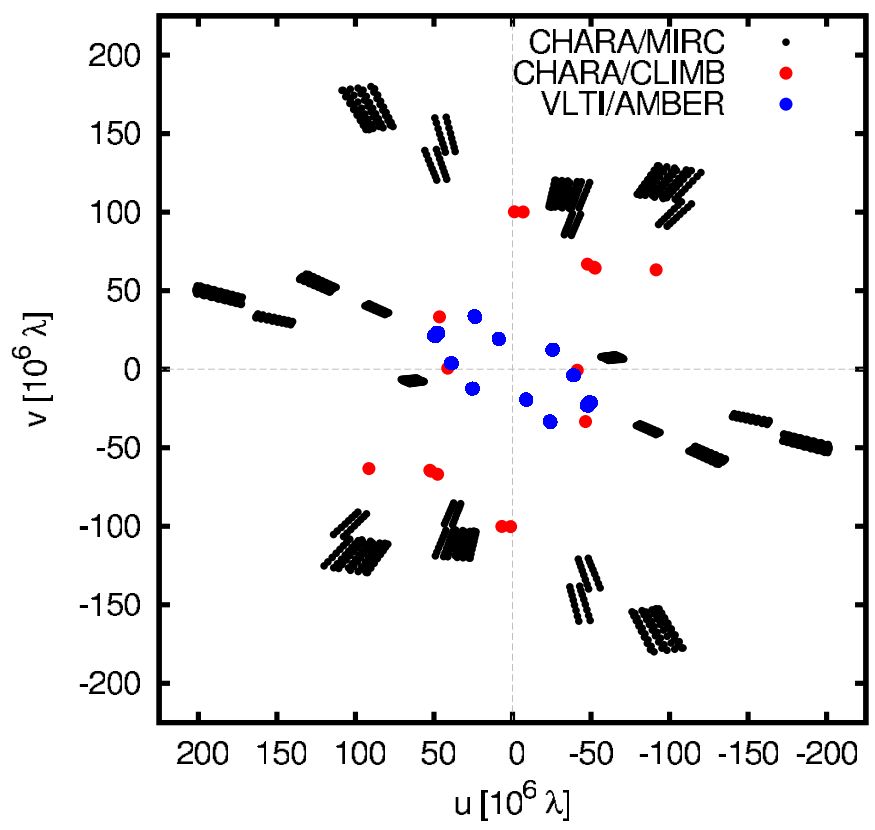

Figure 1. $u v$-coverage achieved with our CHARA/MIRC ( $H$-band), CHARA/CLIMB ( $K$-band), and VLTI/AMBER ( $K$-band) interferometric observations on $\beta$ CMi.

(A color version of this figure is available in the online journal.)

Here, we present near-infrared spectro-interferometric observations on the classical $\mathrm{Be}$ star $\beta \mathrm{CMi}$ with a high spectral resolution of $R=12,000$ in the hydrogen $\mathrm{Br} \gamma$-line, enabling us to constrain the rotation profile directly. In addition, we observed the classical Be star $\zeta$ Tau for the first time in multiple hydrogen line transitions ( $\mathrm{Br} \gamma$ and Pfund lines), providing direct information about the excitation structure within the disk.

In the following, we present our CHARA and VLTI interferometric observations (Section 2). The observations in spectral lines are then first interpreted using a model-independent photocenter analysis approach (Section 3). In Section 4, we present our continuum and kinematical modeling on $\beta \mathrm{CMi}$, followed by our discussion of the results on $\zeta$ Tau (Section 5). Finally, we summarize our findings in Section 6.

\section{OBSERVATIONS}

Our near-infrared $H$-band continuum observations on $\beta \mathrm{CMi}$ were obtained using the CHARA array (ten Brummelaar et al. 2005), which is operated by Georgia State University. The MIRC beam combiner (Monnier et al. 2006b) allowed us to combine the light from four of the six CHARA $1 \mathrm{~m}$ telescopes simultaneously, yielding baseline lengths of up to $330 \mathrm{~m}$, with good baseline coverage (Figure 1). The MIRC data cover the $H$-band with low spectral dispersion $(R=35)$ and was reduced using the University of Michigan MIRC data reduction pipeline (Monnier et al. 2007).

In order to investigate the $K$-band disk geometry of $\beta \mathrm{CMi}$, we employed the CHARA/CLIMB 3 telescope beam combiner (Sturmann et al. 2010). Visibilities and closure phases were derived using the "redclimb" and "reduceir" software. Besides the statistical errors, we also add a calibration uncertainty of 0.05 for the derived visibilities, which represents an empirical value for the typical scatter in the instrument transfer function.

Spectro-interferometric observations with medium (MR mode, $R=1500$ ) and high spectral dispersion (HR mode, $R=12,000)$ were obtained with the Very Large Telescope

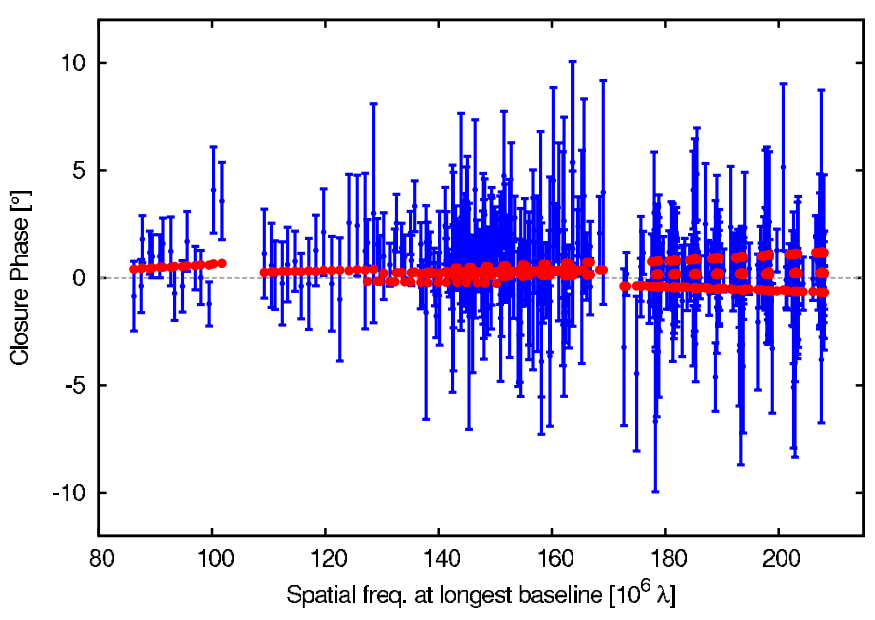

Figure 2. Closure phases measured with the MIRC beam combiner on $\beta \mathrm{CMi}$ (blue data points), overplotted with the model predictions from our best-fit $H$-band model (red data points; Section 4.2).

(A color version of this figure is available in the online journal.)

Interferometer (VLTI) of the European Southern Observatory and the AMBER 3 telescope beam combiner instrument (Petrov et al. 2007). The AMBER observations on $\beta$ CMi were recorded using three $8.2 \mathrm{~m}$ unit telescopes (2009 December 31) and three $1.8 \mathrm{~m}$ auxiliary telescopes (2010 April 23), respectively. The atmospheric piston was stabilized using the FINITO fringe tracker (Le Bouquin et al. 2008), which allowed us to use long detector integration times (DITs) of $1 \mathrm{~s}$ and $6 \mathrm{~s}$ and to record data with high spectral dispersion around the hydrogen $\mathrm{Br} \gamma$-line $\left(\lambda_{\mathrm{Br} \gamma}^{\mathrm{vacuum}}=2.166078 \mu \mathrm{m}\right)$. Due to the presence of residual phase jitter, the absolute visibility calibration of the AMBER data is not reliable, while the important wavelength-differential observables are not affected. Spectra and wavelength-differential visibilities and phases (DPs; Figure 3) were derived from the AMBER data using the amdlib (V3.0) data reduction software (Tatulli et al. 2007; Chelli et al. 2009). The wavelength calibration was done using atmospheric telluric features close to the $\mathrm{Br} \gamma$-line (yielding an accuracy of approximately 1 spectral channel) and by applying a heliocentric-barycentric system correction using heliocentric velocities of $+5.97 \mathrm{~km} \mathrm{~s}^{-1}$ (2009 December 31) and -28.61 $\mathrm{km} \mathrm{s}^{-1}$ (2010 April 23), respectively. For the systemic velocity we assume $+22.0 \mathrm{~km} \mathrm{~s}^{-1}$ (Duflot et al. 1995).

From all CHARA and VLTI interferometric observations, we also derived closure phases (CPs). Both the $H$ - and $K$-band continuum closure phases are consistent with zero on a $2 \sigma$-level, which leads us to conclude that the brightness distribution does not show significant indications for deviations from centrosymmetry. The most constraining CPs have been recorded with MIRC in the $H$-band, which are shown in Figure 2. Our AMBER HR measurements from 2009 December 31 provide us also with a $\mathrm{CP}$ measurement in the $\mathrm{Br} \gamma$-line of $\beta \mathrm{CMi}$, while the derived CPs from the 2010 April 23 dataset are rather noisy and are therefore not included for our model fits.

$\zeta$ Tau was observed on 2010 January 1 using AMBER's MR-mode covering the upper $K$-band $(2.12-2.46 \mu \mathrm{m})$. The assumed systemic velocity for $\zeta$ Tau is $+21.8 \mathrm{~km} \mathrm{~s}^{-1}$ (Duflot et al. 1995). In the $\zeta$ Tau data, we detected not only the $\mathrm{Br} \gamma$ transition (7-4), but also hydrogen Pfund transitions (Figure 4, 1st row), including clear detection of Pf14 (19-5, $2.4477 \mu \mathrm{m})$ to Pf22 $(27-5,2.3591 \mu \mathrm{m})$. Higher Pfund transitions are also present in the spectrum, but cannot be clearly separated due 
Table 1

Observation Log of Our CHARA and VLTI Observations

\begin{tabular}{|c|c|c|c|c|c|c|c|}
\hline Target & $\begin{array}{l}\text { Date } \\
\text { (UT) }\end{array}$ & Instrument & $\begin{array}{l}\text { Spectral } \\
\text { Mode }\end{array}$ & NP & $\begin{array}{l}\text { DIT } \\
\text { (s) }\end{array}$ & $\begin{array}{c}\text { Telescope } \\
\text { Configuration }\end{array}$ & Calibrator(s) \\
\hline \multirow[t]{9}{*}{$\beta \mathrm{CMi}$} & 2009 Dec 31 & AMBER & HR-K 2.172 & 1 & 1 & UT1-UT2-UT4 & HD 71095 \\
\hline & 2010 Apr 23 & AMBER & HR-K 2.172 & 1 & 6 & $\mathrm{~K} 0-\mathrm{G} 1-\mathrm{A} 0$ & HD 71095 \\
\hline & 2008 Dec 2 & MIRC & H35 & 1 & $\cdots$ & S1-E1-W1-W2 & HD 25490, HD 79469, HD 97633 \\
\hline & 2009 Nov 10 & MIRC & H35 & 3 & $\cdots$ & S1-E1-W1-W2 & HD 43042 \\
\hline & 2010 Nov 2 & MIRC & H35 & 3 & $\cdots$ & S1-E1-W1-W2 & HD 43042 \\
\hline & 2010 Dec 14 & MIRC & H35 & 1 & $\cdots$ & S1-E2-W1-W2 & HD 79469, HD 97633 \\
\hline & 2010 Nov 30 & CLIMB & $\mathrm{K}$ & 2 & $\cdots$ & S2-W1-W2 & HD 73262 \\
\hline & 2010 Dec 2 & CLIMB & $\mathrm{K}$ & 1 & $\cdots$ & S1-E2-W1 & HD 73262 \\
\hline & 2010 Dec 3 & CLIMB & $\mathrm{K}$ & 4 & $\cdots$ & S2-E2-W2 & HD 73262 \\
\hline$\zeta \mathrm{Tau}$ & 2010 Jan 1 & AMBER & MR-K 2.3 & 1 & 0.2 & UT1-UT2-UT4 & HD 71095 \\
\hline
\end{tabular}

Notes. Column 5 (NP) denotes the number of pointings obtained on the science star. For the calibrators, we assume the following uniform disk (UD) diameters: for HD 25490 (0.536 \pm 0.037 mas), HD 43042 (0.62 \pm 0.05 mas), HD 71095 (2.009 \pm 0.139 mas), and HD 73262 (0.47 \pm 0.03 mas) we use the diameter estimates from searchCal (Bonneau et al. 2006). The UD diameters of HD 79469 (0.471 \pm 0.027 mas) and HD 97633 (0.68 \pm 0.06 mas) have been estimated by averaging the estimates from three independent photometric methods (Barnes et al. 1978; Bonneau et al. 2006; Kervella \& Fouqué 2008).

to the wide, double-peaked profile of the individual lines. For $\zeta$ Tau, the derived CPs are too noisy to provide additional information and are therefore not included in our further analysis.

In order to search for similar Pfund lines on $\beta \mathrm{CMi}$, we also obtained an AMBER MR-K 2.3 measurement on this star (2010 December 5), but did not detect other emission lines besides $\mathrm{Br} \gamma$. The $\mathrm{Br} \gamma$-line in this MR-measurement shows very similar visibility and DP signatures as our two HR-K measurements, but with lower spectral resolution, and is therefore not included for our further analysis.

Details about the observational setup for all interferometric observations are listed in Table 1. Each observation on a science star was accompanied by observations on interferometric calibrators, allowing us to monitor and correct for the atmospheric and instrumental transfer function.

\section{PHOTOCENTER ANALYSIS}

Differential phases provide unique information about smallscale (sub-mas) photocenter displacements between the blueand red-shifted line wings. These displacements provide a very sensitive measure of the gas kinematics on scales of a few stellar radii. In a first analysis step, we reconstruct the on-sky twodimensional photocenter displacement from the measured DPs by solving the system of linear equations:

$$
\vec{p}=-\frac{\phi_{i}}{2 \pi} \cdot \frac{\lambda}{\vec{B}_{i}},
$$

where $\phi_{i}$ is the differential phase measured on baseline $i, \vec{B}_{i}$ is the corresponding baseline vector, and $\lambda$ is the central wavelength (Le Bouquin et al. 2009). The derived photocenter plots for $\beta \mathrm{CMi}$ and $\zeta$ Tau are shown in Figures 3 and 4 (middle panel), respectively, and clearly reveal rotation-dominated velocity fields for both objects, as indicated by the linear alignment of the photocenter vectors for the different gas velocities and the opposite sign of the photocenter displacement for the blue- and red-shifted emission. In order to associate the DP with the onsky orientation, it is necessary to calibrate the sign of the DP measurements. For this purpose, we employed a VLTI/AMBER reference data set of a binary star with a $\mathrm{Br} \gamma$-emitting circumprimary component, which allows us to derive the correct phase sign from the displacement between the line and continuum photocenter (details will be presented in Kraus et al., in preparation).

For $\beta \mathrm{CMi}$, we determine the position angles $\theta$ for the disk rotation plane at the two epochs to be $138.3 \pm 1.5$ (2009 December 31) and 141.7 \pm 1.4 (2010 April 22). The photocenter vectors corresponding to our highest signal-to-noise observation (2009 December 31) show an interesting arc-like structure in the red-shifted line wing (Figure 3, bottom left), where the photocenter vectors corresponding to low gas velocities are above the derived disk plane, while the high velocities are displaced in the opposite direction. Although the significance of this pattern is still only marginal in our data, we speculate that this pattern might result from opacity effects, with the more distant parts of the disk appearing fainter than the disk parts facing the observer. Such an obscuration screen would displace the photocenter perpendicular to the disk plane, where the amplitude of the displacement is stronger for lower gas velocities, since the low-velocity emission is distributed over a more extended region. Accordingly, the displacement would be strongest at zero velocities, and then symmetrically decrease toward higher velocities. The superposition of this weak displacement (perpendicular to the disk plane) with the displacement due to Keplerian rotation (parallel to the disk plane) might result in the observed arc-shaped structure, which would also provide a unique tool to determine the orientation of the disk in space and the disk rotation sense. In the case of $\beta \mathrm{CMi}$, this implies that the northeastern part of the disk is facing toward the observer (based on the displacement of the low-velocity channels in this direction) and that the disk is in clockwise rotation (based on the location of the red-shifted photocenter displacements in the northwestern quadrant).

Another intriguing feature in the $\beta \mathrm{CMi}$ data is the $W$-shaped profile, which we observe in the wavelengthdependent visibilities and DP on our longest interferometric baselines (Figure 3, 2nd and 3rd rows). Likely, this profile indicates that the visibility function of the line-emitting region passes through a visibility null and transits from the first to the second visibility lobe. Since this effect would reverse the direction of the photocenter vector, we manually correct the phase sign in these corresponding spectral channels close to the line center. For $\zeta$ Tau, we can determine the rotation axis for the $\mathrm{Br} \gamma$ 

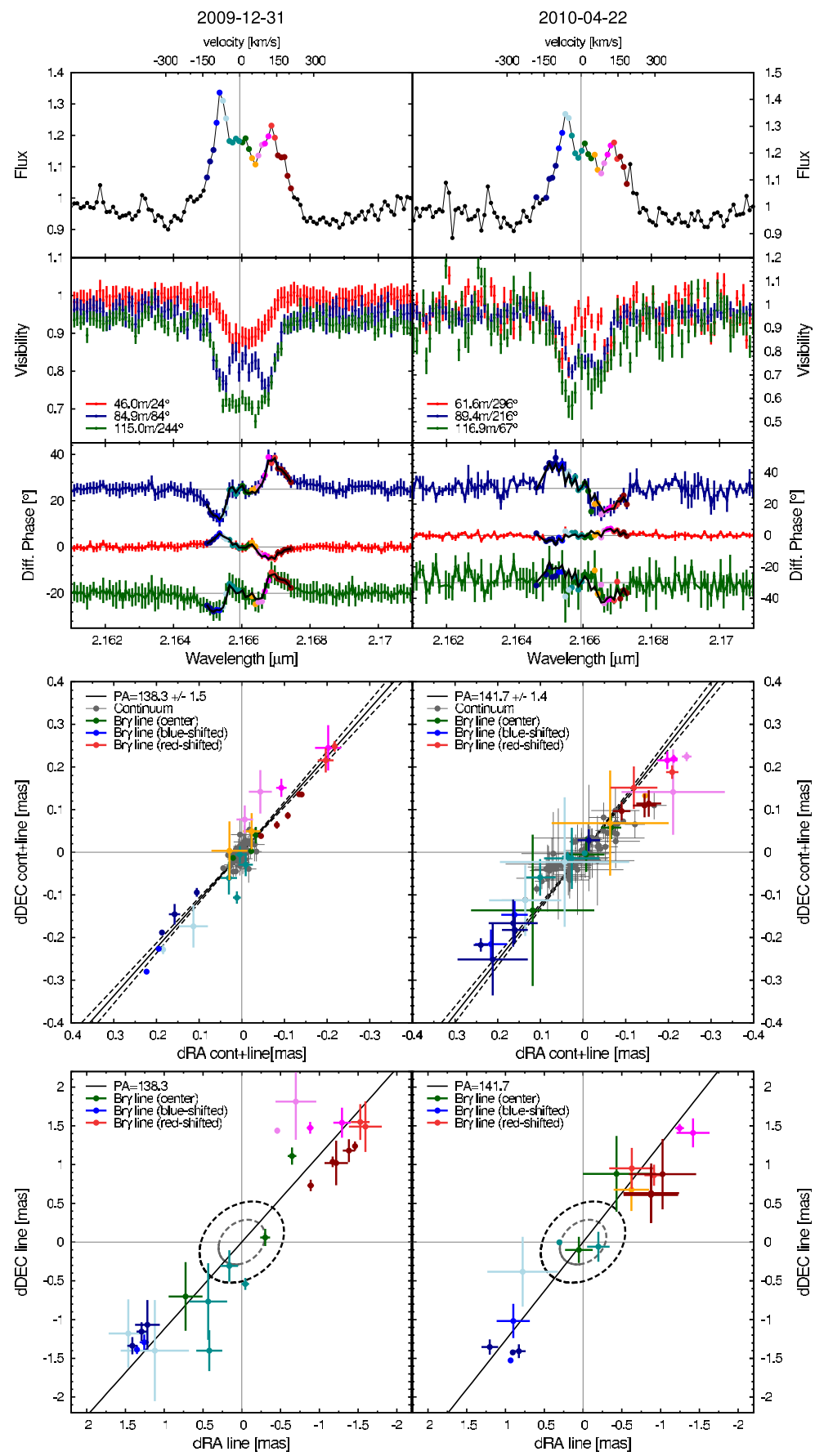

Figure 3. Upper panel: VLTI/AMBER spectra (1st row), visibilities (2nd row), and DPs (3rd row) measured on $\beta$ CMi for the epochs 2009 December 31 (left) and 2010 April 22 (right). Middle panel: from the measured DPs, we derive for each spectral channel the two-dimensional photocenter displacement vector (including continuum and line contributions; east is plotted left and north is up). The flux and DP data points as well as the derived photocenter vectors have been color-coded based on the Doppler velocity (see the upper panel to relate each color to a wavelength). The DPs corresponding to the astrometric solutions are shown in the middle panel as solid black lines. Bottom panel: using the procedure outlined in Section 3, we corrected for the continuum contributions in the line spectral channels, revealing the photocenter displacement corresponding to the line emission only. Besides the determined photocenter offsets, for comparison we also show the size of the $H$-band continuum-emitting disk (black ellipse, FWHM Gaussian, Section 4.2) and of the stellar photosphere (gray ellipse, Section 4.1).

(A color version of this figure is available in the online journal.)

$(123.9 \pm 2.0)$ and the nine Pf14-Pf22 transitions (127.6 \pm 1.5$)$ separately and find that the line-emitting gas rotates in the same disk plane within the observational uncertainties of $\sim 2^{\circ}$.

Using the aforementioned procedure, it is possible to reliably measure the direction of the photocenter displacement, while the length of the displacement vector is biased by the contributions from the underlying continuum emission. In order to remove these contributions from the measured observables $(F, V, \phi)$, we apply the method outlined by Weigelt et al. (2007) and interpolate the continuum flux $\left(F_{c}\right)$ and continuum visibility $\left(F_{c}\right)$ from the adjacent continuum. The visibility and DP of the pure line emitting-region $\left(V_{l}, \phi_{l}\right)$ are then given by

$$
\begin{gathered}
\left|F_{l} V_{l}\right|^{2}=|F V|^{2}+\left|F_{c} V_{c}\right|^{2}-2 \cdot F V \cdot F_{c} V_{c} \cdot \cos \phi \\
\sin \phi_{l}=\sin \phi \frac{|F V|}{\left|F_{l} V_{l}\right|},
\end{gathered}
$$



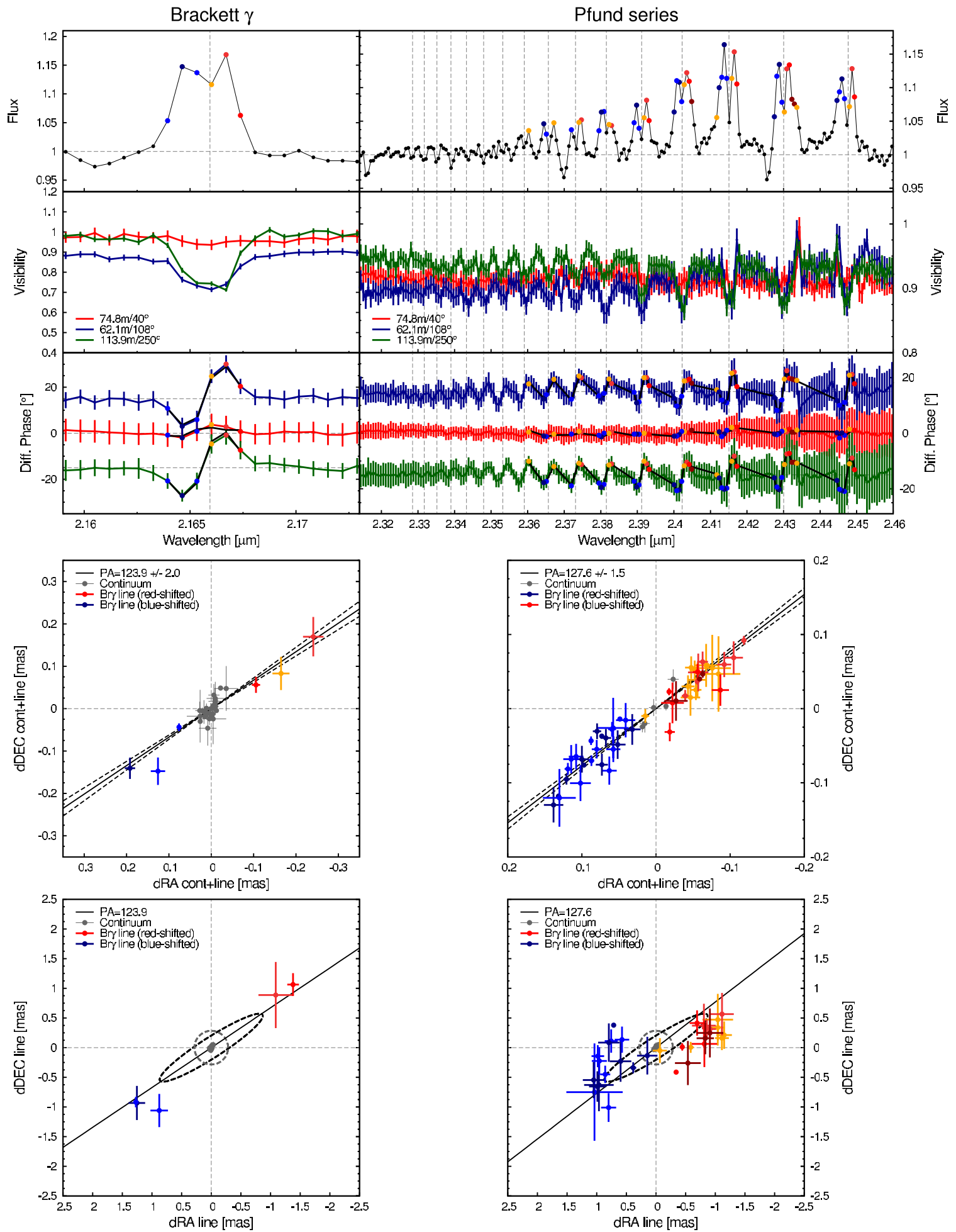

Figure 4. Upper panel: VLTI/AMBER spectra (1st row), visibilities (2nd row), and DPs (3rd row) measured on $\zeta$ Tau for the epoch $2010 \mathrm{January} 1 \mathrm{in}$ the Br $\gamma$ (left) and Pf14-22 transitions (right). Middle/bottom panel: photocenter displacement vectors derived from the measured DPs (including line and continuum emission, middle panel) and the continuum-corrected DPs (tracing the line emission only, bottom panel). For comparison, we also show the size of the $H$-band continuum-emitting disk, as determined by Schaefer et al. (2010) for epoch 2009 November 10 (black ellipse, FWHM Gaussian) and of the stellar photosphere (gray ellipse).

(A color version of this figure is available in the online journal.)

where $F_{l}=F-F_{c}$ denotes the flux contribution from the spectral line. The continuum-corrected DPs are then used to derive the photocenter displacement of the pure line-emitting region (Figures 3 and 4, bottom panel). Applying this correction will provide the real centroid offset of the line emission, but also introduce noise from the visibility and flux measurements, resulting in an increased scatter in the position angle distribution. Therefore, we decided to measure the position angle of the rotation axis from the uncorrected line+continuum photocenter displacements (Figures 3 and 4, middle panel), while the 


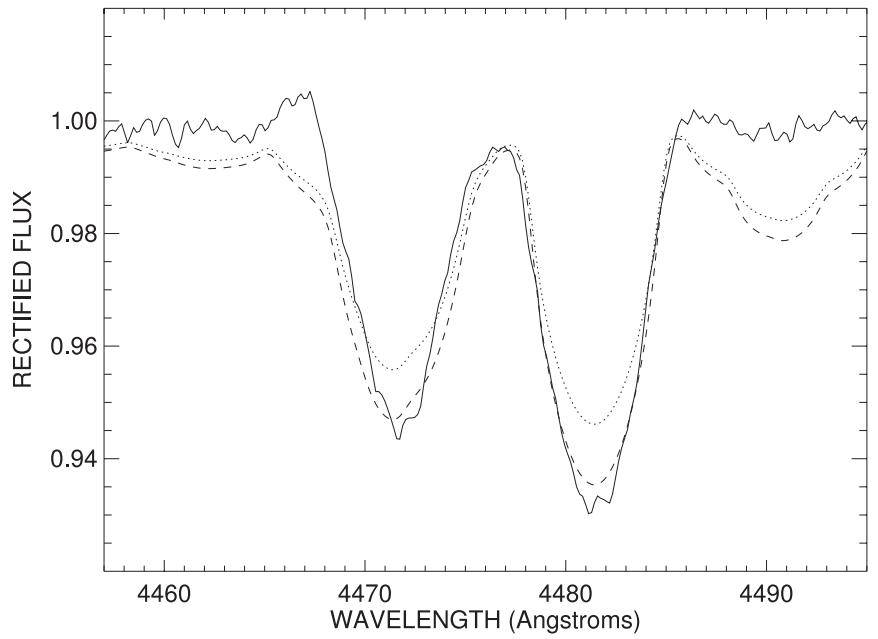

Figure 5. Spectrum of the He I $447.1 \mathrm{~nm}$ and $\mathrm{Mg}$ II $448.1 \mathrm{~nm}$ line of $\beta$ CMi (solid line). The dashed line shows a photospheric model without disk contributions, while the dotted line corresponds to a model where $20 \%$ disk continuum contributions have been added.

continuum-corrected photocenter displacements (bottom panel) will be used later on to construct a position-velocity diagram and to compare the spatial origin in different line tracers.

\section{DISCUSSION ON $\beta$ CANIS MINORIS}

$\beta$ CMi (HR 2845) is a relatively quiet B8V-type classical Be star located at a distance of $52.2_{-2.2}^{+2.4}$ pc (Tycner et al. 2005). Ground-based photometric monitoring has provided no clear indication of significant variability (Pavlovski et al. 1997), while there is some marginal evidence for long-term variations in the H $\alpha$-profile (Pollmann 2002; Hesselbach 2009).

In the following, we present a refined model for the photospheric emission of $\beta \mathrm{CMi}$ taking the near-critical stellar rotation into account (Section 4.1), followed by our modeling of the interferometric data in continuum emission (Section 4.2). Given that the disk rotation signatures in the $\mathrm{Br} \gamma$ emission line are overlaid on the rotation signatures of the star (in $\mathrm{Br} \gamma$ absorption), we investigate the influence of the stellar rotation on our measurements (Section 4.3). In the following, we construct a position-velocity diagram (Section 4.4) and present a full kinematical modeling in Section 4.5.

\subsection{Constraining the Stellar Parameters}

In order to obtain a model for the photospheric emission of $\beta \mathrm{CMi}$, we searched for a consistent set of stellar parameters, taking the evidence for near-critical rotation into account (Saio et al. 2007). For this purpose, we employed our rapid rotator code (Monnier et al. 2007; Che et al. 2011), which simulates the stellar oblateness and surface temperature distribution using the modified von Zeipel theorem (gravity darkening coefficient $=0.188$ ), and computes model images in the continuum and in the photospheric $\mathrm{Br} \gamma$ absorption line.

In order to constrain the stellar parameters, we computed a small parameter grid, in which we systematically varied the inclination angle $i$ and the fractional angular velocity $\omega / \omega_{\text {crit }}$ (where $\omega_{\text {crit }}$ is the critical angular velocity). For a given inclination angle and fractional angular velocity, we start our iterative process by assuming a stellar mass $M_{\star}$, from which we compute the polar radius $R_{\text {pole }}$ using the rotation velocity $v \sin i=244 \pm 6 \mathrm{~km} \mathrm{~s}^{-1}$ (Yudin 2001). The polar temperature $T_{\text {pole }}$ is derived from the $V$-band magnitude $(V=2.89)$. Any
Table 2

Grid of Stellar Parameters, Obtained for $\beta$ CMi Using Our Fast Rotator Model (Section 4.1)

\begin{tabular}{lccccccccc}
\hline \hline Model & $i$ & $\omega / \omega_{\text {crit }}$ & $\begin{array}{c}M_{\star} \\
\left(M_{\odot}\right)\end{array}$ & $\begin{array}{c}R_{\text {pole }} \\
\left(R_{\odot}\right)\end{array}$ & $\begin{array}{c}R_{\text {eq }} \\
\left(R_{\odot}\right)\end{array}$ & $\begin{array}{c}T_{\text {pole }} \\
(\mathrm{K})\end{array}$ & $\begin{array}{c}P \\
(\text { days })\end{array}$ & $\begin{array}{c}L^{\text {app }} \\
\left(L_{\odot}\right)\end{array}$ & $\begin{array}{c}T_{\text {eff }}^{\text {app }} \\
(\mathrm{K})\end{array}$ \\
\hline A & 45 & 0.99 & 3.70 & 3.32 & 4.61 & 13200 & 0.676 & 267 & 11558 \\
B & 45 & 0.96 & 4.11 & 3.03 & 3.94 & 14600 & 0.577 & 343 & 13085 \\
C & 45 & 0.93 & 4.47 & 2.87 & 3.59 & 15650 & 0.527 & 413 & 14235 \\
D & 40 & 0.99 & 3.98 & 2.95 & 4.10 & 14550 & 0.546 & 337 & 12871 \\
E & 40 & 0.96 & 4.49 & 2.74 & 3.56 & 16050 & 0.475 & 435 & 14505 \\
F & 40 & 0.93 & 4.91 & 2.60 & 3.25 & 17300 & 0.433 & 532 & 15847 \\
G & 35 & 0.99 & 4.43 & 2.62 & 3.64 & 16200 & 0.433 & 437 & 14447 \\
H & 35 & 0.96 & 5.03 & 2.44 & 3.17 & 18100 & 0.377 & 590 & 16474 \\
\hline
\end{tabular}

disk continuum flux in the optical band will tend to dilute the photospheric spectrum causing absorption lines to appear weaker than expected. We checked for such line reduction in blue spectra of $\beta$ CMi obtained by Grundstrom (2007). Figure 5 shows the observed profiles (solid line) of He I $447.1 \mathrm{~nm}$ and Mg II $448.1 \mathrm{~nm}$ based upon the average of 11 spectra made between 2005 and 2009 with the Kitt Peak National Observatory Coude Feed Telescope (spectral resolution $R=12,500$ ). The observed spectrum is compared with a theoretical flux spectrum (dashed line) from the grid of LTE models by Rodríguez-Merino et al. (2005). We adopted average parameters for the visible hemisphere of the star of $T_{\text {eff }}=11,800 \mathrm{~K}, \log g=3.8$, and $V \sin i=230 \mathrm{~km} \mathrm{~s}^{-1}$ (Frémat et al. 2005), and the model spectrum was convolved with a simple rotational broadening function for a linear limb darkening coefficient of $\epsilon=0.42$ (Wade \& Rucinski 1985). Note that this approach assumes a spherical star, ignores gravity darkening, and neglects changes in the local intensity spectrum with orientation, but these simplifications are acceptable for our purpose of checking for systematic line depth differences. We see that the match of the $\mathrm{Mg}$ II $\lambda 4481$ profile is satisfactory, but the wings of the He I $\lambda 4471$ line appear too high, probably due to low level emission in this transition. Adding a $20 \%$ disk flux contribution and renormalizing the spectrum yields a diluted version (dotted line), which appears to be much too weak compared with the observed spectrum. This comparison suggests that the disk continuum emission in the blue range is negligible, and thus we will ignore any disk contribution to the optical flux in the following discussion.

With the derived polar radius and bolometric luminosity, we are able to locate the stellar position on the HR diagram, after correcting for the rotational effect (Che et al. 2011). The stellar mass from the HR diagram is used as initial value for the next iteration step, until convergence between the assumed mass and the mass estimated from the HR diagram is reached.

For each parameter combination, our model provides the rotation period, apparent luminosity $L^{\text {app }}$, and apparent effective temperature $T_{\text {eff }}^{\text {app }}$, as listed in Table 2. Comparing the model effective temperature and luminosity with the observational constraints for $\beta \mathrm{CMi}\left(T_{\text {eff }}=12,050 \mathrm{~K} ; L=195 \pm 60 L_{\odot}\right.$; Saio et al. 2007), we find good agreement for models A, B, and D. Due to its consistency with the inclination and effective temperature (Sections 4.2 and 4.5), we favor model D, suggesting $i=40^{\circ}$, $\omega / \omega_{\text {crit }}=0.99, T_{\text {pole }}=14,550, v \sin i=244 \mathrm{~km} \mathrm{~s}^{-1}$, $T_{\mathrm{eff}}^{\mathrm{app}}=12871 \mathrm{~K}$, and a polar and equatorial radius of $R_{\text {pole }}=$ $2.95 R_{\odot}=0.26 \mathrm{mas}$ and $R_{\mathrm{eq}}=4.10 R_{\odot}=0.36 \mathrm{mas}$, respectively. We plot the values corresponding to our model grid in the HR diagram shown in Figure 6 and compare it to the 


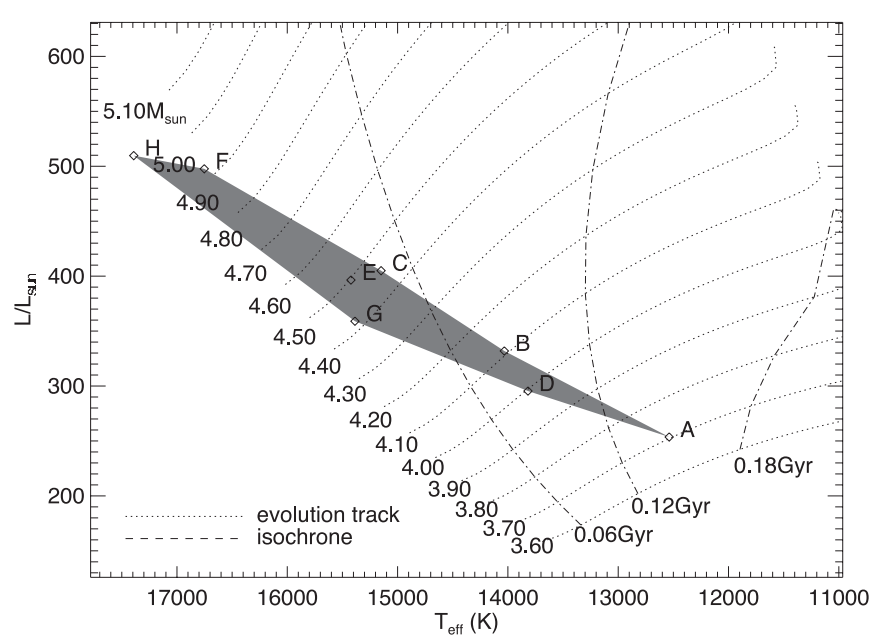

Figure 6. Hertzsprung-Russell diagram, including the solutions from our small rapid rotator grid for $\beta \mathrm{CMi}$ (Table 2). The numbers on the left side give the stellar mass of the corresponding evolution track, while the numbers at the bottom gives the evolutionary age corresponding to the shown isochrones (Yi et al. 2003; Demarque et al. 2004). As outlined in Section 4.1, only models A, B, and D satisfy the observational constraints on the stellar effective temperature. Furthermore, model D satisfies best our inclination obtained with interferometry $\left(38.5 \pm 1^{\circ}\right)$

evolutionary tracks from Yi et al. (2003) and Demarque et al. (2004), yielding an evolutionary age of $\sim 0.1-0.15$ Gyr.

Obviously, an important input parameter for our modeling procedure is the rotation velocity $v \sin i$, for which we use an average value from the literature. Townsend et al. (2004) argued that most $v \sin i$ measurements on Be stars might systematically underestimate the true projected rotation value due to the effect of gravity darkening. In order to test this scenario, we have artificially increased the measured $v \sin i$-value by $10 \%$ and repeated our modeling procedure. We find that the increase in $v \sin i$ results in a significant increase in the stellar mass, apparent effective temperature, and apparent luminosity, making the model prediction much less consistent with the observed values. Therefore, we suggest that in the case of $\beta \mathrm{CMi}$, the $v \sin i$-measurements are not significantly biased by gravity darkening, which is likely a result of the intermediate inclination angle of $\beta \mathrm{CMi}$ (the effect would be stronger for an equator-on viewing angle) and the use of our moderate gravity darkening law $\left(T_{\text {eff }} \propto g_{\text {eff }}^{0.188}\right.$, Che et al. 2011), which is likely more realistic than the classical von Zeipel darkening coefficient $\left(T_{\text {eff }} \propto g_{\text {eff }}^{0.25}\right)$ adopted by Townsend et al. (2004). Clearly, the accurate determination of $v \sin i$ remains a fundamental problem for constraining the stellar parameters of near-critical rotating stars. Therefore, we are currently working on incorporating the computation of model spectra in our modeling procedure, which will then be fitted to observed spectra together with the photometric and interferometric constraints (Che et al., in preparation).

The modeling provides spectra as well as model images in the continuum emission and in hydrogen photospheric absorption lines, which we will use as a representation of the stellar brightness distribution for our modeling of the disk in the following sections.

\subsection{Continuum Disk Geometry}

With projected baseline lengths up to $B=314 \mathrm{~m}$, our CHARA/MIRC interferometric observations allow us to constrain the disk geometry in eight spectral channels in the $H$-band with an effective resolution of $\lambda / 2 B=0.5$ mas. A commonly applied modeling approach is to approximate the disk emission with a Gaussian intensity profile, which is superposed on a uniform disk representing the stellar surface (e.g., Tycner et al. 2005; Meilland et al. 2009; Pott et al. 2010; Schaefer et al. 2010). A caveat of this approach is that a significant part of the disk emission is concentrated at radii $r<R_{\star}$, and therefore wrongly attributed to the disk instead of the stellar flux. Accordingly, these models will systematically overestimate the disk emission with respect to the stellar emission $\left(F_{\text {disk }} / F_{\star}\right)$, resulting in inconsistencies with spectral energy distribution fitting results. In order to avoid these biases, we employ an elliptical Gaussian model, where the radial intensity profile at $r>R_{\star}$ is given by a half-Gaussian, resulting in a proper estimation of the $F_{\text {disk }} / F_{\star}$ ratio. As alternative model, we considered an inclined ring model, where the radial intensity profile is given by a Gaussian centered at radius $a$ from the star, with a fixed fractional width of $25 \%$ (see Monnier et al. 2006a for details). Based on radiative transfer simulations of the brightness distribution in classical Be star disks (e.g., Waters 1986), we consider the Gaussian model a better representation of the expected emission profile in a $\mathrm{Be}$ disk, while the ring model is more suited for comparison with results from the literature (e.g., Meilland et al. 2006).

For all models, the stellar photosphere is represented by the aforementioned rapid rotator model (Section 4.1) and the position angle $\theta$ of the stellar equator and the disk major axis are aligned. Besides the disk major axis $a$ (ring major radius or Gaussian half-width half maximum), the PA $\theta$, and the inclination $i$, we also treat the flux ratio between the stellar and disk component $\left(F_{\text {disk }} / F_{\star}\right)_{H}$ as a free parameter. The model was fitted to the MIRC squared visibilities, closure phases, and triple amplitudes using a least-square fitting procedure, resulting in the best-fit model shown in Figure 7. The best-fit model parameters are displayed in Table 3, including $1 \sigma$-errors, which we have determined using a boot strapping procedure. We also tested whether the agreement can be improved using a skewed ring model (Monnier et al. 2006a), but find that introducing any disk asymmetry only marginally improves the fit. Our model includes minor asymmetries due to the brightened polar region in our rapid rotator model, which results in small closure phases ( $\lesssim 1.2)$, consistent with the measurement (Figure 2).

The determined disk position angles of 139.2 (ring model) and 139.3 (Gaussian model) are in excellent agreement with the gas disk rotation axis determined with our VLTI/AMBER photocenter analysis $(140.0 \pm 1.7)$.

Using the detailed information about the $H$-band geometry obtained with our extensive MIRC data set, we then fitted our Gaussian model to the CLIMB data to determine the $K$-band continuum geometry. Given the lower amount of observational constraints for the $K$-band, we treated only the disk-to-star flux ratio $\left(F_{\text {disk }} / F_{\star}\right)_{K}$ as a free parameters and kept the remaining parameters fixed. The resulting best-fit visibility curves are shown in Figure 7 . With $\left(F_{\text {disk }} / F_{\star}\right)_{K}=0.25_{-0.08}^{+0.09}$, we do not find any significant deviations between the $K$-band and $H$-band flux ratio.

\subsection{Investigating the Differential Phase Signatures of the Stellar Rotation in the Bry Absorption Line}

With the currently achievable accuracy, DP measurements can already reveal photocenter displacements two to three orders smaller than the formal angular resolution $(\lambda / 2 B)$. For instance, our 2009 December 31 AMBER observations exhibit a DP accuracy of $\sim 0.8$ (standard deviation over all continuum 

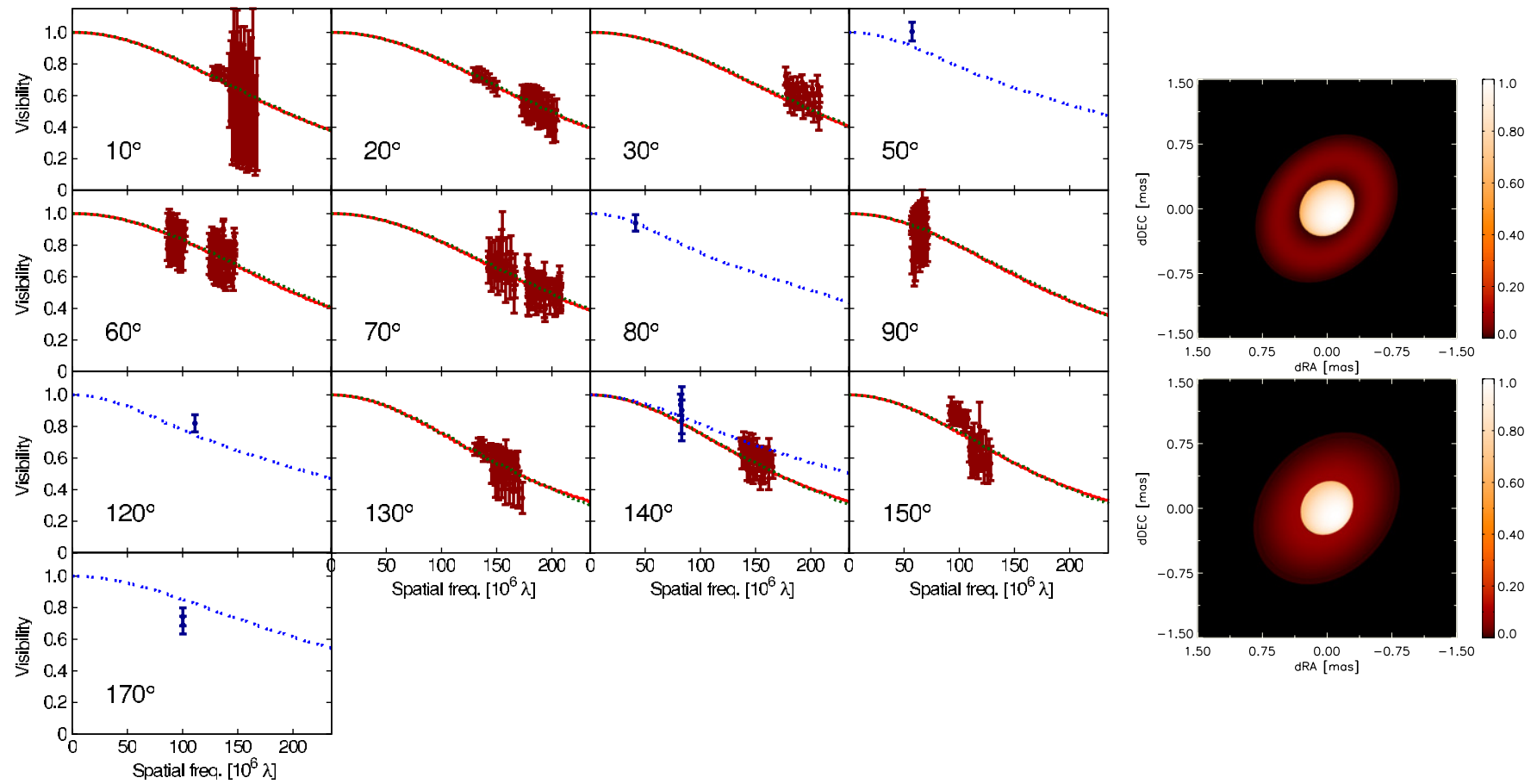

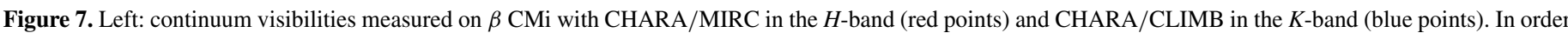

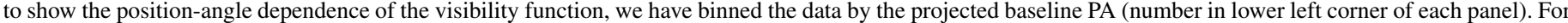

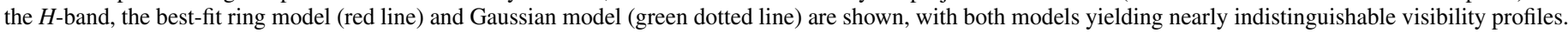

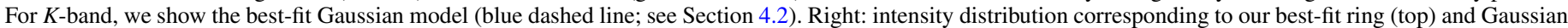
model (bottom).

(A color version of this figure is available in the online journal.)

Table 3

Model Fitting Results for the Disk of $\beta$ CMi

\begin{tabular}{|c|c|c|c|c|c|c|c|}
\hline \multirow[t]{2}{*}{ Parameter } & & & \multicolumn{2}{|c|}{$\begin{array}{c}\text { Continuum } \\
H \text {-band }\end{array}$} & \multirow{2}{*}{$\begin{array}{c}\text { Continuum } \\
K \text {-band } \\
\text { (Gaussian model) }\end{array}$} & \multicolumn{2}{|c|}{$\begin{array}{c}\text { Line } \\
\text { Brackett } \gamma\end{array}$} \\
\hline & & & (Ring model) & (Gaussian model) & & (Keplerian rot.) & (Free rotation law) \\
\hline Flux ratio & $F_{\text {disk }} / F_{\star}$ & & $0.16_{-0.03}^{+0.07}$ & $0.18_{-0.03}^{+0.05}$ & $\begin{array}{l}0.20_{-0.15}^{+0.15} \\
\end{array}$ & $\cdots$ & $\cdots$ \\
\hline Major axis size & $a$ & (mas) & $1.19_{-0.24}^{+0.41(b)}$ & $0.33_{-0.14}^{+0.18}$ & $0.33^{(\mathrm{a}),(\mathrm{c})}$ & $\cdots$ & $\cdots$ \\
\hline Position angle ${ }^{(e)}$ & $\theta$ & $\left({ }^{\circ}\right)$ & $138.2_{-7.8}^{+3.1}$ & $139.5_{-6.3}^{+4.4}$ & $139.5^{(\mathrm{c})}$ & $136.8^{(\mathrm{c})}$ & $136.8^{(\mathrm{c})}$ \\
\hline Inclination $^{(\mathrm{d})}$ & $i$ & $\left({ }^{\circ}\right)$ & $39.9_{-6.4}^{+7.2}$ & $40.1_{-9.2}^{+5.9}$ & $40.1^{(\mathrm{c})}$ & $38.5 \pm 1$ & $38.5^{(\mathrm{c})}$ \\
\hline Outer disk radius & $R_{\text {out }}$ & (mas) & $\cdots$ & $\cdots$ & $\cdots$ & $5.8 \pm 0.2$ & $5.8^{(\mathrm{c})}$ \\
\hline Stellar mass & $M_{\star}$ & $\left(M_{\odot}\right)$ & $\ldots$ & $\ldots$ & $\ldots$ & $3.5 \pm 0.2$ & $3.5^{(\mathrm{c})}$ \\
\hline Rotation law index & $\beta$ & & $\ldots$ & $\cdots$ & $\ldots$ & $-0.5^{(\mathrm{c})}$ & $-0.5 \pm 0.1$ \\
\hline Rotation velocity & $f_{\mathrm{kep}}(1 \mathrm{AU})$ & & $\cdots$ & $\ldots$ & $\cdots$ & $1.0^{(\mathrm{c})}$ & $1.0 \pm 0.1$ \\
\hline Radial intensity index & $q$ & & $\cdots$ & $\cdots$ & $\cdots$ & $-1.6 \pm 0.2$ & $-1.6^{(\mathrm{c})}$ \\
\hline & $\chi_{r}^{2}$ & & 1.77 & 1.77 & 1.17 & 1.66 & 1.66 \\
\hline
\end{tabular}

Notes. (a) For the Gaussian model, we give the half-width half-maximum (HWHM) of the half-Gaussian intensity profile, measured along the major axis. (b) For the ring models, we give the ring major axis diameter. (c) This parameter has been kept fixed throughout the modeling procedure (see Sections 4.2 and 4.5 for details). (d) An inclination angle of $i=0$ denotes a face-on disk orientation. (e) Throughout this paper, all position angles are measured along the disk major axis and east of north.

spectral channels), corresponding to a photocenter displacement of $\sim 8$ microarcsecond ( 0.008 mas) on the employed $115 \mathrm{~m}$ baseline. Given that this is much smaller than the equatorial stellar diameter $(\sim 720$ microarcsecond), it is important to investigate whether the measured DP signatures might also contain contributions from the photospheric $\mathrm{Br} \gamma$ absorption line, which is tracing the stellar rotation and is underlying the Br $\gamma$-line emission.

In order to simulate the effect of stellar rotation on our interferometric observables, we employ our rapid rotator code and compute the stellar surface brightness distribution around the $\mathrm{Br} \gamma$-line with a similar resolution as our AMBER observations (Figure 8, top). For this, we align the stellar rotation axis with the measured rotation axis $(\theta=227.5$, Section 3$)$. $\mathrm{Br} \gamma$ absorption on one side of the photosphere will shift the photocenter toward the opposite direction. Taking this into account, we adjust the orientation of our model photosphere, so that the $\mathrm{Br} \gamma$ absorption in the blue-shifted line wing matches the measured direction of the photocenter displacement at redshifted wavelengths. In addition to the photospheric emission, we include disk emission in our model, assuming $\left(F_{\text {disk }} / F_{\star}\right)_{K}=$ 0.25, as determined in Section 4.2. 

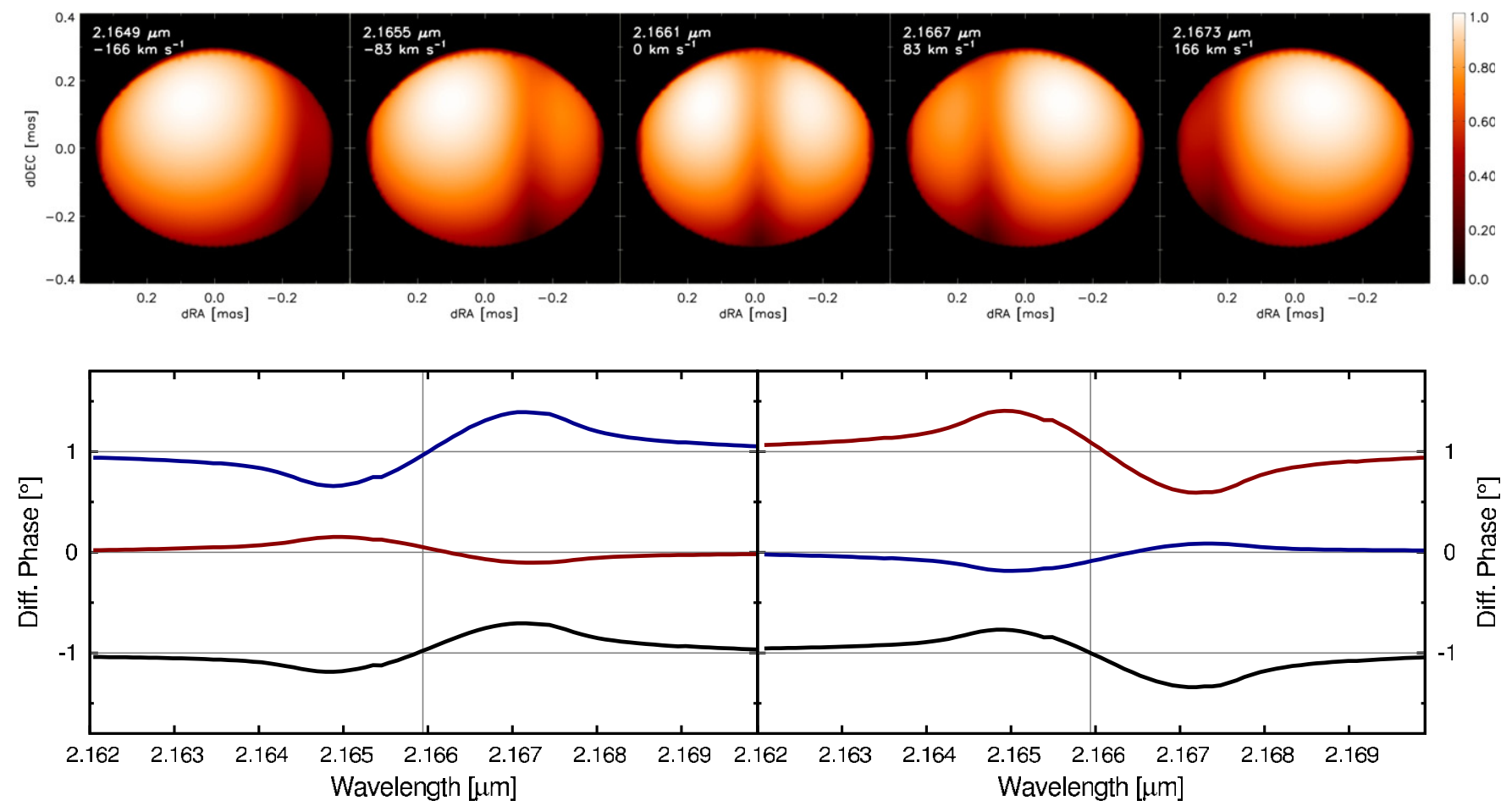

Figure 8. Top: model images from our rapid rotator model for $\beta \mathrm{CMi}$ for some representative wavelengths (Section 4.3). Bottom: model differential phases (including the stellar photosphere only) computed for the baselines employed by our $\beta$ CMi VLTI measurements from 2009 December 31 (left) and 2010 April 23 (right).

(A color version of this figure is available in the online journal.)

From the model, we compute the corresponding DPs and find signatures $\phi \lesssim 0.5$ on all baselines (Figure 8, bottom). This result reflects the fact that the fraction of the photospheric absorption to the total $K$-band emission is rather small $(<8 \%)$ and that the absorption appears as a rather diffuse structure on the extended stellar surface. We conclude that the photocenter signatures due to stellar rotation (in photospheric absorption lines) are a minor effect compared to the rotation signatures of the disk (in line emission), but nevertheless result in DP signatures comparable to the currently achievable DP accuracy and are therefore important, in particular for more detailed future studies.

\subsection{Constraints on the Disk Kinematics from the Position-Velocity Diagram}

Position-velocity $(p-v)$ diagrams provide a powerful tool for the interpretation of a disk velocity field and are commonly employed in radio astronomy to derive the rotation profile of circumnuclear galactic disks or protostellar disks using, for instance, maser (e.g., Miyoshi et al. 1995; Pestalozzi et al. 2009) or molecular emission tracers (e.g., Sofue \& Rubin 2001; Isella et al. 2007). Using our photocenter displacement measurements, we can construct an equivalent diagram from our VLTI interferometric data by measuring the length of the continuum-corrected photocenter displacement vectors projected on the disk plane $\theta=140.0$. Performing this projection on the disk plane also allows us to avoid a potential bias due to opacity effects, since these effects would move the photocenter only perpendicular to the disk plane (Section 3). The resulting $p-v$ diagram (Figure 9) shows a symmetric rotation curve, which we interpret using the simple model of a thin Keplerian-rotating disk (Weintroub et al. $2008)$. The disk extends from an inner $\left(R_{\text {in }}\right)$ to an outer $\left(R_{\text {out }}\right)$ radius and the line-of-sight (LOS) velocity $v$ of the emission element located at radius $r$ and at angle $\vartheta$ in the disk plane is given by

$$
v_{\mathrm{kep}}(r, \vartheta)=\sqrt{\frac{G M_{\star}}{r}} \sin \vartheta \cdot \cos i,
$$

where $\vartheta$ is measured against the LOS and $G$ is the gravitational constant. Considering only the emission from the outermost disk annulus (i.e., at $R_{\text {out }}$ and $\vartheta=-\pi \ldots+\pi$ ) will result in a straight line in the $p-v$ diagram (line $A-B$ in Figure 9). Adding the emission from the remaining disk annuli $\left(R_{\text {in }}\right.$ to $R_{\text {out }}$ ) will result in a characteristic "bowtie"-shaped filled region in the $p-v$ diagram, such as commonly observed in $\mathrm{CO}$ imaging observations (Sofue \& Rubin 2001; Isella et al. 2007). However, astrometric observations (such as radio masers or photocenter displacements) trace the light barycenter of each annulus, corresponding to the $B-C$ and $A-D$ curves in Figure 9 (for more details see Weintroub et al. 2008). We fit this simple model to the $\beta$ CMi $p-v$ diagram assuming a fixed inclination of $i=40^{\circ}$ (as determined in Section 4.2), which yields best agreement for $M_{\star}=3.6 \pm 0.3 M_{\odot}, R_{\text {out }}=2.4 \pm 0.2 \mathrm{mas}$, and $R_{\text {in }}<1.0$ mas (solid curve in Figure 9).

\subsection{Detailed Modeling of the Disk Kinematics}

By reducing the information content to purely astrometric data, the $p-v$ diagram analysis method presented in the last section provides a very intuitive and powerful method to constrain the gas velocity field in the observed line tracer. In this section, we will use a more sophisticated model in order to make full use of the rich information contained in our spectrointerferometric observations, including spectra, wavelengthdifferential visibilities, DPs, and CPs. The $\mathrm{Br} \gamma$-emitting gas is assumed to be located in a thin disk plane, which is justified due to the expected small opening angle of Be star disks (e.g., 


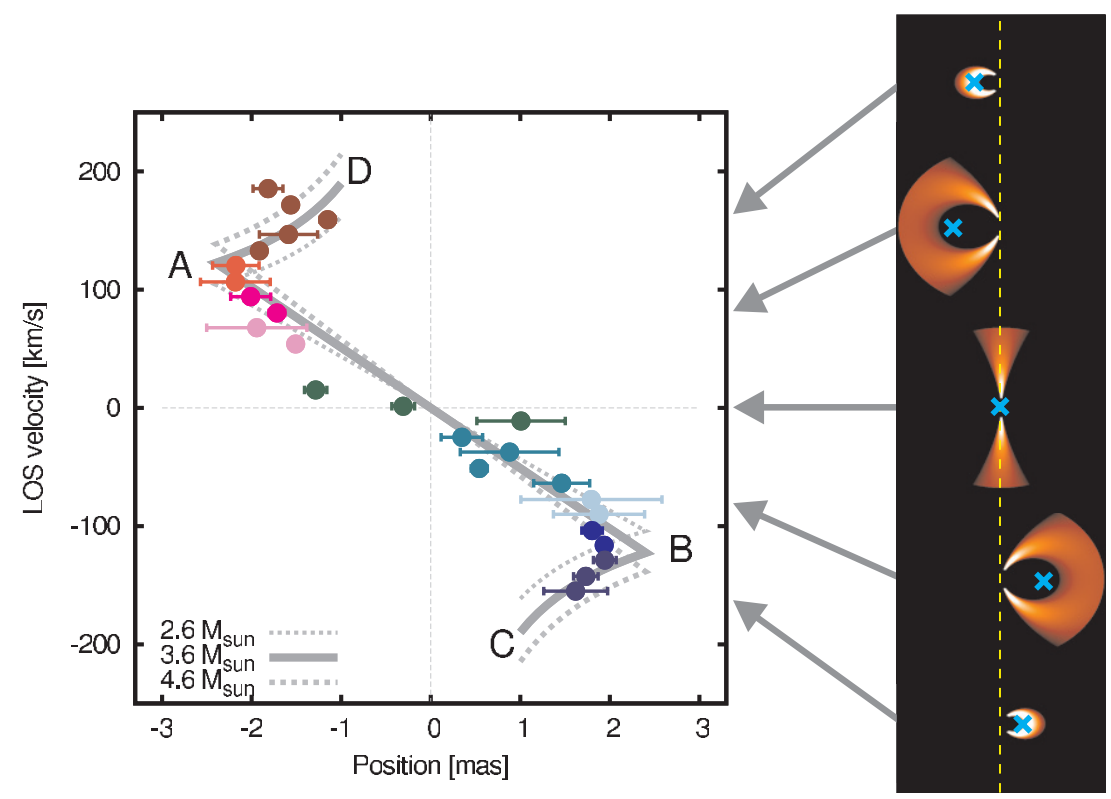

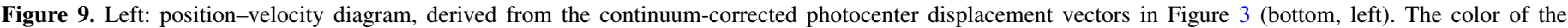

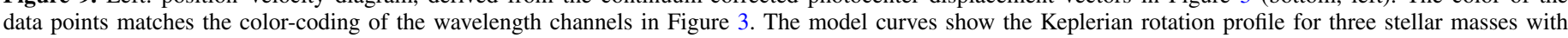

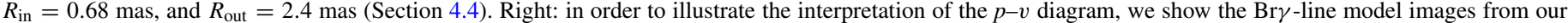

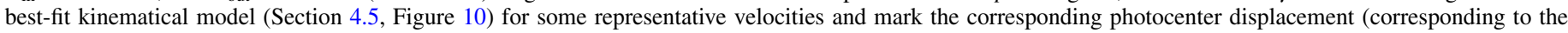

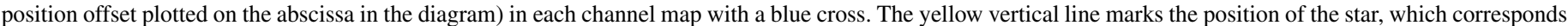
to the photocenter of the continuum emission and the zero position in the $p-v$ diagram.

(A color version of this figure is available in the online journal.)

Bjorkman \& Cassinelli 1993) and the intermediate inclination angle under which $\beta \mathrm{CMi}$ is observed (Section 4.2).

The gas kinematics is parameterized with a rotation profile $|\vec{v}(r)|=f_{\text {kep }}\left(R_{\text {ref }}\right) \cdot\left(r / R_{\text {ref }}\right)^{\beta}$, where $\beta=-1$ for rotation with constant angular momentum, $\beta=-0.5$ for Keplerian rotation, $\beta=0$ for constant rotation, and $\beta=+1$ for solid body rotation (e.g., Stee 1996). $f_{\text {kep }}=\left|\vec{v}\left(R_{\text {ref }}\right)\right| /\left|\vec{v}_{\text {kep }}\left(R_{\text {ref }}\right)\right|$ is the orbital velocity at the reference radius $R_{\mathrm{ref}}=1 \mathrm{AU}$, expressed as fraction of the Keplerian velocity $\left|\vec{v}_{\text {kep }}(r)\right|=\left(G M_{\star} / r\right)^{-1 / 2}$. In the model, the line emission extends from the stellar surface to an outer radius $R_{\text {out }}$ with a radial power-law intensity profile $I_{l}(r) \propto r^{q}$. To include thermal line broadening in our kinematic model, we adopt a constant radial gas temperature of $0.6 \cdot T_{\text {eff }}$ $(=7860 \mathrm{~K}$ for $\beta \mathrm{CMi})$, as suggested by the radiative transfer modeling from Carciofi \& Bjorkman (2006).

We include both line and continuum emission in our model and compute the interferometric observables for our given VLTI array configurations and the covered wavelength channels. The wavelength-dependent model visibilities and phases are then fitted to the VLTI interferometric data using a reduced $\chi_{r}^{2}$ goodness-of-fit estimator (see Kraus et al. 2009 for a definition), including our flux, visibility, DP, and CP constraints.

For the continuum emission, we assume the geometry determined with our CHARA observations (Section 4.2) and use this model to renormalize the AMBER continuum visibilities. In order to incorporate the underlying photospheric $\mathrm{Br} \gamma$ absorption, we include the photosphere model discussed in Section 4.3, although, as discussed above, the influence on the differential phase ( $\lesssim 0.5$ on all baselines) is still within the measurement uncertainties.

The disk position angle is fixed to $\theta=140.0$, as determined by our model-independent photocenter analysis (Section 3 ). The remaining six parameters in our modeling are the outer disk radius $R_{\text {out }}$, the inclination $i$, the stellar mass $M_{\star}$, the radial intensity power-law index $q$, the disk rotation index $\beta$, and the velocity at the reference radius $R_{\text {ref }}$ (expressed as fraction of the Keplerian velocity, $\left.f_{\text {kep}}\right)$. In a first step, we fix the velocity profile to Keplerian rotation $(\beta=-0.5$, $f_{\text {kep }}=1$ ) and vary the remaining four parameters systematically on a grid, yielding the best-fit model shown in Figure 10. The corresponding parameters and uncertainties are listed in Column 4 of Table 3 . In a second step, we test also non-Keplerian velocity fields, yielding the best-fit values listed in Column 5 . The resulting $\chi^{2}$-surfaces are shown in Figures 11 and 12 and the uncertainties have been derived using the bootstrapping technique.

Our model fits show that the intriguing phase inversion observed at the line center on our longest VLTI baselines (Figure 10, 3rd row) can be explained with the phase jumps in the Fourier phase crossing a visibility null. These phase jumps appear in the same spectral channels where we measure visibility minima in the $W$-shaped visibility profile, indicating that the visibility function of the pure line-emitting geometry transits here from the first to the second visibility lobe (Figure 10, 2nd and 3rd row). At the same time, the continuum emission is only marginally resolved, which results in the measured composite line+continuum visibility with a rather high contrast of $\sim 0.7$. The phase jumps and visibility minimums are a basic property of the Fourier transform of strongly resolved objects and are reproduced very naturally and without fine-tuning from our kinematical modeling. Therefore, our results do not support the idea outlined by Štefl et al. (2011) that these features might indicate secondary dynamical effects or polar mass outflows.

As best-fit value for the radial intensity index, we yield $q=-1.6 \pm 0.2$. Isothermal viscous decretion disk models, such as discussed in Bjorkman \& Carciofi (2005), predict a radial disk surface density law $\Sigma(r) \propto r^{-2}$, corresponding to $q=-2$ in the 

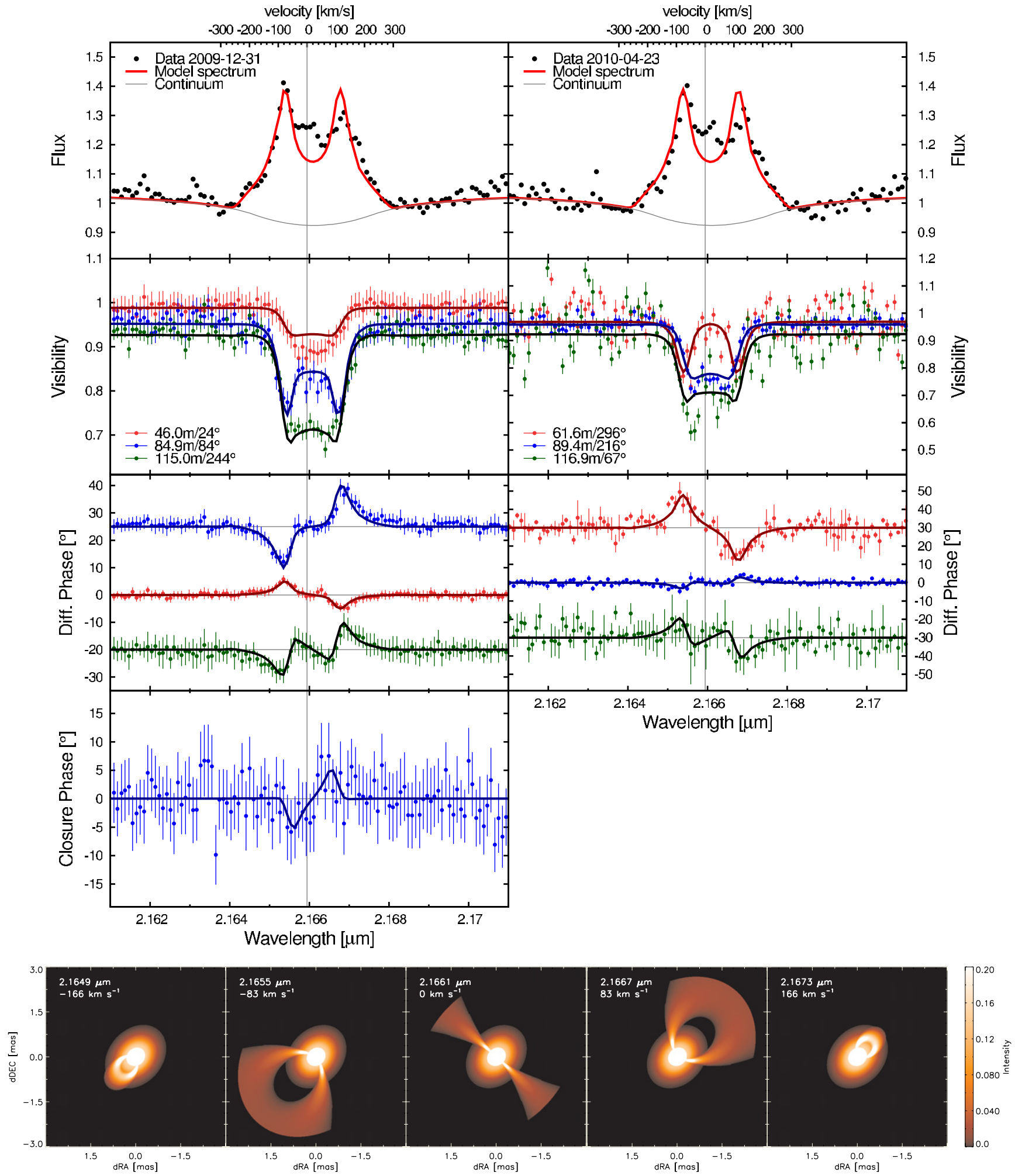

Figure 10. Upper panel: comparison of the VLTI/AMBER $\beta$ CMi spectra (1st row), visibilities (2nd row), DPs (3rd row), and CPs (4th row) with our Keplerian disk model (solid lines). The three different colors correspond to the three baselines, where the associated projected baseline lengths and PAs are labeled in the 2nd row. Lower panel: to illustrate our kinematical model, we show intensity channel maps for five representative wavelengths.

(A color version of this figure is available in the online journal.)

optically thin case. More realistic non-LTE disk models suggest a more shallow surface density profile $\left(\Sigma(r) \propto r^{-2 \ldots-1}\right)$ due to a steep temperature drop in the inner few stellar radii of the disk (Carciofi \& Bjorkman 2008). Therefore, we conclude that our measured intensity profile is well consistent with these models.
The most significant deviation of our simple kinematical model from the data is in the precise shape of the $\mathrm{Br} \gamma$-spectrum. For instance, the measured spectra show a weak emission component in the line center (Figure 10), which is not reproduced by the model and which might be related either to 


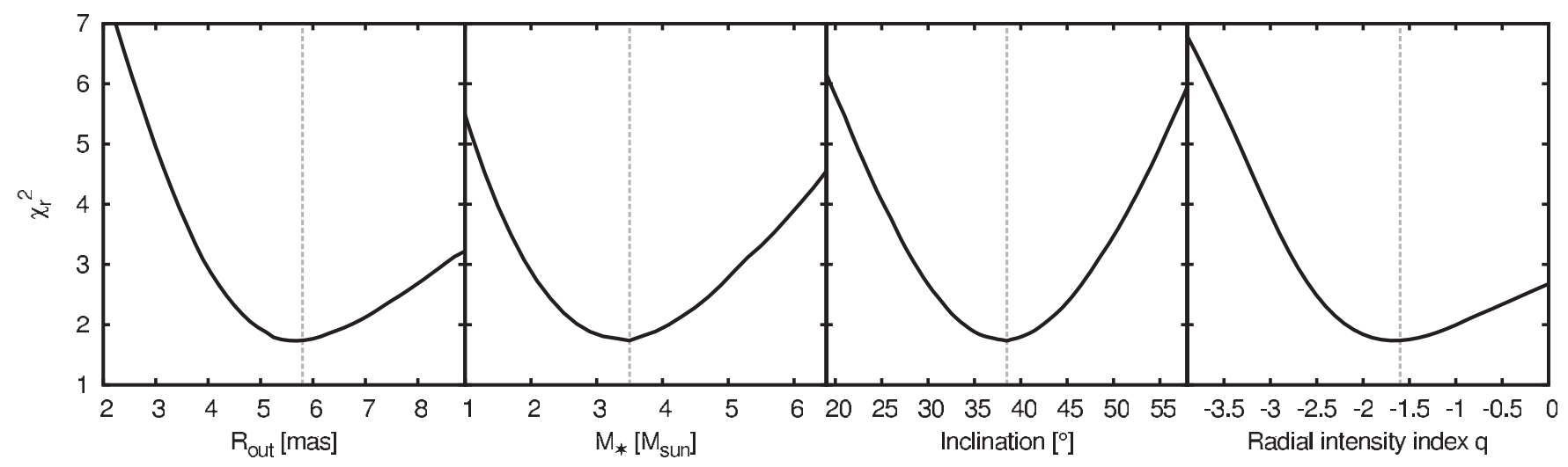

Figure 11. $\chi^{2}$-surface derived from our $\beta$ CMi model grid (Section 4.5) around the best-fit solution assuming a Keplerian velocity field (Table 3).

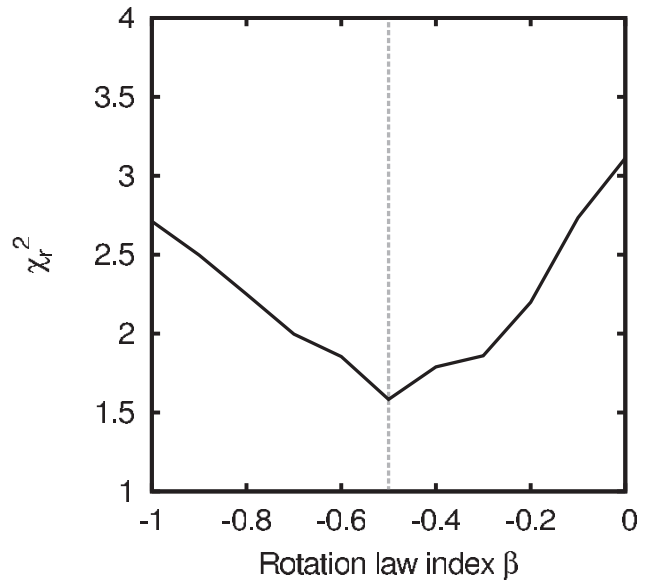

Figure 12. $\chi^{2}$-surface for the rotation law index $\beta$ derived from our $\beta \mathrm{CMi}$ model grid including also non-Keplerian velocity fields (Section 4.5).

the presence of a uniformly distributed low-velocity gas halo or, more likely, to radiative transfer effects.

Another deviation between the model and the observation concerns the weak $V / R$-asymmetry which can be observed in our VLTI/AMBER Br $\gamma$ spectra at both epochs (Figure 3, top row) and which is not reproduced by our axialsymmetric kinematical model. Within the 113 days covered by our AMBER observations, no changes in the $V / R$ asymmetry could be observed, which is consistent with the conclusion by Tycner et al. (2005) and Jones et al. (2011) that the $\mathrm{H} \alpha$ profile does not show significant long-term variability. It is interesting to compare the average photocenter displacement between the blue- and red-shifted line emission $(2.5$ mas $)$ with the characteristic size of the $\mathrm{H} \alpha$-emitting region (2.13 mas, Tycner et al. 2005), which suggests that $\mathrm{Br} \gamma$ emerges from a similar spatial region as $\mathrm{H} \alpha$ and a much more extended region than the $H$ - and $K$-band continuum emission $\left(R_{\mathrm{Br} \gamma} \sim R_{\mathrm{H} \alpha}>R_{\text {cont }}\right.$, Figure 3), in agreement with the prediction from Carciofi (2010). However, a detailed comparison is difficult due to the nonsimultaneity of the different observations.

\section{DISCUSSION ON $\zeta$ TAURI}

$\zeta$ Tau is a particularly well-studied classical Be star which shows a cyclic variability in the flux ratio of the violet- and redshifted wing of the double-peaked $\mathrm{H} \alpha$ emission line (Rivinius et al. 2006; Štefl et al. 2009). These $V / R$ variations exhibit a period of $\sim 1429$ days and are generally attributed to a global one-armed density oscillation in a Keplerian (or nearly Keplerian) disk (Okazaki 1991).

A kinematic model for the $\mathrm{Br} \gamma$-line of some earlier AMBER MR observations on $\zeta$ Tau was presented by Štefl et al. (2009) and Carciofi et al. (2009) and suggested a Keplerian rotation profile. Our spectro-interferometric observations on $\zeta$ Tau provide a $\operatorname{Br} \gamma$ measurement at a new epoch in the $\mathrm{H} \alpha V / R$-phase and cover, for the first time, also the wavelength region around the hydrogen Pfund lines. Besides our $\mathrm{Br} \gamma$ and Pfund series data, we include $\mathrm{H} \alpha$-sizes from the literature, namely the GI2T photocenter measurement by Vakili et al. $\left(1998, \sim 7 R_{\star}\right.$ or $\sim 1.33$ mas) and the Mark III and Navy Prototype Optical Interferometer (NPOI) measurements by Quirrenbach et al. (1994, 1997) and Tycner et al. (2004). It is important to note that the Mark III and NPOI results were based on visibility amplitudes instead of differential phases. Also, a direct comparison with these earlier observations is complicated by the known $\mathrm{V} / \mathrm{R}$ variability of $\zeta$ Tau, although we note that our measurement at phase 0.484 is reasonably close to the $\mathrm{H} \alpha$-measurement at phase 0.577 , presented by Tycner et al. (2004). We convert the Gaussian FWHM derived by these studies to photocenter displacements by computing the centroid of the corresponding Gaussian brightness distributions. We find that $\mathrm{Br} \gamma$ emerges from a similar spatial region than $\mathrm{H} \alpha$, but a more extended region than the near-infrared continuum emission, as already found for $\beta \mathrm{CMi}$ (Section 4.5). The Pfund emission originates from intermediate stellocentric radii $\left(R_{\mathrm{H} \alpha} \sim R_{\mathrm{Br} \gamma}>R_{\mathrm{Pf}}>R_{\text {cont }} ;\right.$ Figure 4$)$.

\subsection{Signatures of the Known One-armed Oscillation}

Both in Br $\gamma$ and in the Pf14-Pf22 lines, we detect a doublepeaked line profile and clear rotation signatures in the differential phases. All line transitions exhibit a photocenter displacement with a stronger amplitude in the southeastern (blue-shifted) than in the northwestern (red-shifted) lobe (Figure 4, middle panel). Such an asymmetric displacement is consistent with the presence of a one-armed oscillation in the disk (Štefl et al. 2009; Carciofi et al. 2009). CHARA/MIRC observations by Schaefer et al. (2010) showed that the one-armed oscillation pattern can also be observed as an asymmetry in the $H$-band continuum emission. Using multi-epoch data, they find that the position angle of the asymmetry is correlated with the spectroscopic variability and precesses around the star with the $\mathrm{H} \alpha V / R$ period. Our observation (2010 January 1) adds an additional epoch 


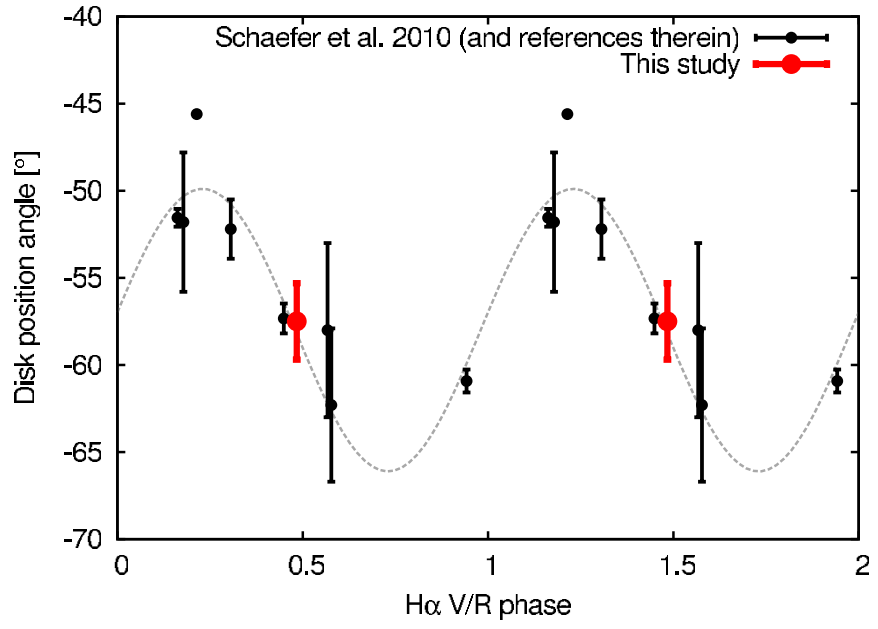

Figure 13. $\zeta$ Tau disk position angle, plotted as function of the $\mathrm{H} \alpha V / R$ phase, including data presented in Schaefer et al. (2010) and in this study. Our new phase measurement (phase 0.484 ) is in good agreement with the disk precession law proposed by Schaefer et al.

(A color version of this figure is available in the online journal.)

for this analysis at the $V / R$ phase 0.484 (assuming maximum phase at JD $=2454505.0$ and a period of 1429 days, Schaefer et al. 2010). We find that the spiral density maximum is located toward the southeast of the central star and the measured PA is consistent with the sinusoidal PA modulation proposed by Schaefer et al. (2010), as shown in Figure 13. Based on our independent calibration of the DP-sign (see Section 3), we find that the approaching (blue-shifted) part of the $\zeta$ Tau disk is located southeast of the star, which corresponds to an opposite rotation sense than the one adopted by Štefl et al. (2009).

The reported polarization angle for $\zeta$ Tau (32.9-33.1, Quirrenbach et al. $1997 ; 35^{\circ} \pm 4^{\circ}$, Ghosh et al. $1999 ; 32^{\circ}-33^{\circ} .5$, McDavid 1999) is in excellent agreement with the rotation axis position angle $(35.8 \pm 2.0)$ found by our spectro-interferometric observations.

\subsection{Probing the Disk Excitation Structure Using Multi-transition Spectro-interferometry}

In order to test whether the measured differences in the stellocentric emitting radius of the $\mathrm{Br} \gamma$ and Pfund transitions are consistent with the expected excitation structure in the disk, we construct a simple radiative transfer model assuming local thermodynamic equilibrium (LTE). We assume that the lineemitting material is located in an equatorial disk which extends outward from the stellar radius with a constant vertical density per unit volume and a half-opening angle of $\Theta=5^{\circ}$. Then, we integrate for each radius $r$ the optical depth $\tau_{v}$ in vertical direction, assuming hydrogen under LTE conditions and an isothermal temperature distributions. The number density for the different excitation levels and ionization stages is computed using the Saha and Boltzmann equation assuming a Gaussian line profile with thermal line broadening (Wilson et al. 2009). The Einstein coefficients for the different hydrogen transitions are estimated using the series expansion published by Omidvar $\&$ McAllister (1995). The radial temperature and surface density profile are parameterized with $T(r)=T_{\text {eff }}\left(r / R_{\star}\right)^{-0.5}$ (Stee \& de Araujo 1994) and $\Sigma(r)=\Sigma_{0}\left(r / R_{\star}\right)^{-2}$ with $\Sigma_{0}=2.1 \mathrm{~g} \mathrm{~cm}^{-2}$ (Carciofi et al. 2009). For the stellar temperature, equatorial stellar radius, and distance, we assume $T_{\text {eff }}=19370 \mathrm{~K}$, $R_{\star}=7.7 R_{\odot}$, and $d=126 \mathrm{pc}$, respectively (Carciofi et al. 2009).

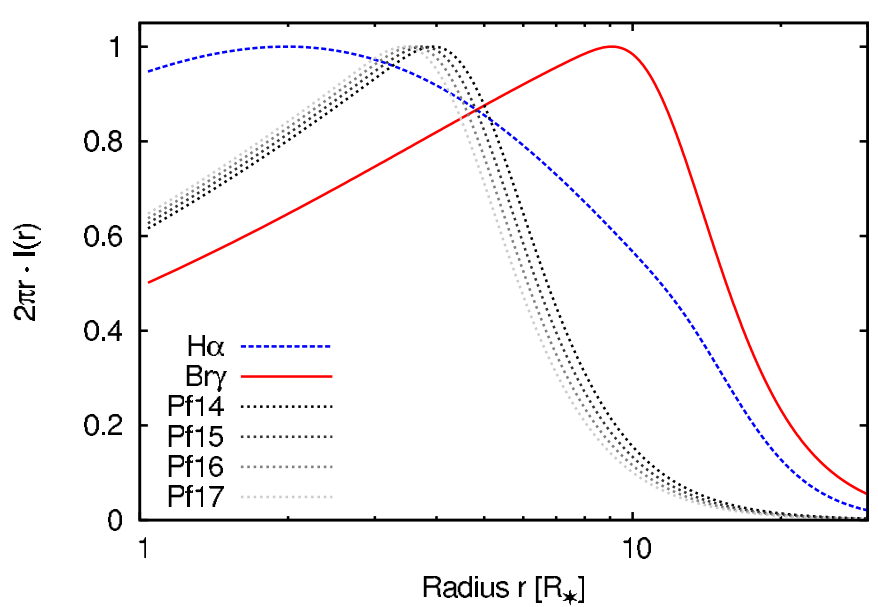

Figure 14. Radial intensity profile, as computed with our LTE model for different line transitions (Section 5.2). The intensity has been weighted by the emitting area and normalized to the peak intensity.

(A color version of this figure is available in the online journal.)

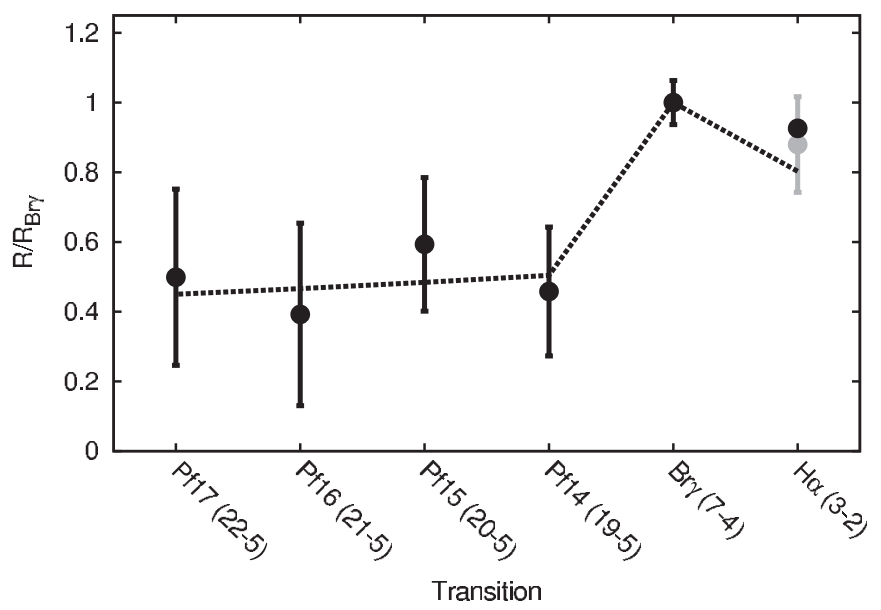

Figure 15. Origin of the $\mathrm{Br} \gamma$ and Pfund line emission from $\zeta$ Tau, as measured by the length of the photocenter displacement vector, averaged over all velocity channels for a given line transition (the error bars represent the standard deviation in the different velocity channels). In addition, we include the $\mathrm{H} \alpha$-line photocenter displacement by Vakili et al. (1998; black data point) and the $\mathrm{H} \alpha$ size estimates from Quirrenbach et al. (1994), Quirrenbach et al. (1997), and Tycner et al. (2004), which we converted from Gaussian FWHM to the corresponding photocenters (gray data point). The dashed line shows the prediction from our LTE excitation model (Section 5.2).

Using the radiative transfer equation for LTE conditions $\left(I_{\nu}(r)=B_{v}(T)\left(1-e^{-\tau_{\nu}(r)}\right)\right)$, we compute the emitted intensity per unit area as function of radius $r$ in the disk, where $B_{v}$ is the Planck spectrum for temperature $T$ at frequency $v$. From the radial intensity profiles (Figure 14) we compute the centroid of the brightness distribution, which is then compared to the stellocentric emission radius measured in the different line transitions. Given that our model assumes a simplified vertical density structure and does not include inclination effects, we do not aim to match the absolute sizes of the emitting region in all line transitions, but focus instead on the relative sizes. For this step, we normalize both the measured and the model photocenter offsets relative to $\mathrm{Br} \gamma$. The comparison between the $\mathrm{H} \alpha, \mathrm{Br} \gamma$, and Pf14-17 relative sizes and our LTE model is shown in Figure 15. We find that we can reproduce important observational features, in particular that

1. $\mathrm{Br} \gamma$ originates from a similar spatial region in the disk as $\mathrm{H} \alpha\left(R_{\mathrm{Br} \gamma} \sim R_{\mathrm{H} \alpha}\right)$. This result is also in agreement with 
the predictions from more sophisticated non-LTE radiative transfer computations, such as made by Carciofi (2010). In order to better characterize the differences between the $\mathrm{Br} \gamma$ and $\mathrm{H} \alpha$-emitting region, contemporaneous observations in these two wavelength bands will be required.

2. $\mathrm{Br} \gamma$ originates from a more extended region than the Pfund lines $\left(R_{\mathrm{Br} \gamma}>R_{\mathrm{Pf}}\right)$. Computing the weighted average of the measurements in the individual transitions, we yield $R_{\mathrm{Pf}} / R_{\mathrm{Br} \gamma}=0.48 \pm 0.12$. For the Pfund lines, we are not aware of earlier predictions obtained with non-LTE radiative transfer codes.

Our observational results confirm the finding from Pott et al. (2010), which found $R_{\mathrm{Br} \gamma}>R_{\mathrm{Pf}}$ on the classical Be star $48 \mathrm{Lib}$, and consolidates their suggestion that the measured size differences can already be explained with the expected optical depth differences between these line transitions. We encourage theoreticians and modelers to employ their more sophisticated non-LTE radiative transfer codes in order to test the influence of inclination and non-LTE effects, although, based on the results from Iwamatsu \& Hirata (2008, e.g., their Figure 3), we expect no significant departure from LTE for the inner disk regions and the high transitions traced by our observations. Future multi-transition spectro-interferometric observations with improved $u v$-coverage might also measure the precise radial intensity profile in a model-independent fashion. Together with sophisticated radiative transfer simulations, these observations will reveal the excitation structure of the disk and constrain parameters such as the temperature profile and the vertical disk structure, which are currently difficult to access.

\section{CONCLUSIONS}

Using CHARA and VLTI interferometry, our study combined high angular resolution (with baseline lengths up to $330 \mathrm{~m}$ ) with kinematical information obtained at high spectral dispersion, yielding direct observational constraints on the gas distribution, excitation structure, and kinematics of the disks around two classical Be stars. Using a model-independent photocenter analysis method we derived the disk rotation axis for the prototypical objects $\beta \mathrm{CMi}$ and $\zeta$ Tau and spatially and spectrally resolved the disk rotation profile on scales of a few stellar radii. For both objects, we find that the determined gas rotation plane agrees well with the orientation of the continuumemitting disk as resolved by CHARA, although there is also clear evidence for substructure in the disk around $\zeta$ Tau, revealing a one-armed oscillation, as indicated by different displacement amplitudes in the blue- and red-shifted line wing.

Using our data set on $\beta \mathrm{CMi}$ we constructed a position-velocity diagram, which can be interpreted using the well-established procedures from radio interferometry, but probes the milliarcsecond scale position displacements resulting from the rotating disk around this Be star. From our kinematical constraints, we derive the dynamical mass of the central star to $3.5 \pm 0.2 M_{\odot}$, which is in excellent agreement with earlier spectroscopic studies (Saio et al. 2007). The inclination of the system is $38.5 \pm 1^{\circ}$, as determined with our CHARA continuum and VLTI line observations. As shown with our detailed kinematical modeling, the rotation law is Keplerian $(\beta=-0.5 \pm 0.1)$ and we do not have to include an expanding velocity component in order to explain our data. Furthermore, our kinematical model allowed us to identify the origin of the phase inversion, which has now been observed in the differential phases in five out of eight Be stars. These phase jumps correspond to the transition from the first to the second visibility lobe, removing the necessity for speculations beyond the canonical star+disk paradigm (Štefl et al. 2011).

For $\zeta$ Tau, we obtained spectro-interferometric observations covering simultaneously the $\mathrm{Br} \gamma$ and at least nine transitions from the Pfund line series. For all transitions, we detect a significantly stronger photocenter displacement in the redshifted line wing than in the blue-shifted line wing, tracing the one-armed oscillation which has been deduced for the $\zeta$ Tau disk before. Comparing the photocenter displacement in the different line transitions, we find that the Pfund, Brackett, and Balmer lines originate from different stellocentric emitting regions $\left(R_{\text {cont }}<R_{\mathrm{Pf}}<R_{\mathrm{Br} \gamma} \sim R_{\mathrm{H} \alpha}\right.$ ), which we can reproduce qualitatively with a simple LTE line radiative transfer model. More work, including non-LTE radiative transfer modeling, will be required in order to derive quantitative constraints.

By detecting a purely Keplerian velocity field, our observations are inconsistent with disk-formation mechanisms incorporating a strong outflowing velocity component, such as the wind compression scenario, which predicts a strong radial velocity component comparable to the escape velocity (Bjorkman $\&$ Cassinelli 1993). On the other hand, our kinematical constraints, as well as the measured hydrogen line intensity profiles (with a radial power law index $q=-1.6 \pm 0.2$ ) are consistent with the predictions from Keplerian viscous decretion disk models (Lee et al. 1991). As shown by Kato (1983) and discussed in various reviews (e.g., Carciofi 2010), viscous Keplerian disks are also able to produce one-armed density oscillations, such as detected for $\zeta$ Tau.

Considering that our kinematic constraints have been obtained using a very limited number of individual measurements (two pointings on $\beta \mathrm{CMi}$, one pointing on $\zeta$ Tau), our study also illustrates the high effectiveness achievable with spectrointerferometry, in particular if a very high spectral resolution is employed or several line transitions are observed.

We thank A. Carciofi for helpful discussions on polarization effects in the infrared, A. Mérand for validating our spectral line imaging code, N. Morrison for providing Ritter spectra, and $\mathrm{C}$. Jones for providing us information about the photometric variability of $\beta \mathrm{CMi}$. We acknowledge the constructive interaction with J.-B. Le Bouguin concerning the phase-sign calibration of VLTI. This work was done in part under contract with the California Institute of Technology (Caltech), funded by NASA through the Sagan Fellowship Program (S.K. is a Sagan fellow). J.D.M. and G.H.S. acknowledge support for this work provided by the National Science Foundation under grants AST-0707927 and AST-1009080. The MIRC beam combiner was developed with funding from the University of Michigan. The CHARA Array is funded by the Georgia State University, by the National Science Foundation through grant AST-0908253, by the W. M. Keck Foundation, by the NASA Exoplanet Science Institute, and the David and Lucile Packard Institute.

Facilities: CHARA, VLTI.

\section{REFERENCES}

Barnes, T. G., Evans, D. S., \& Moffett, T. J. 1978, MNRAS, 183, 285

Bjorkman, J. E., \& Carciofi, A. C. 2005, in ASP Conf. Ser. 337, The Nature and Evolution of Disks Around Hot Stars, ed. R. Ignace \& K. G. Gayley (San Francisco, CA: ASP), 75

Bjorkman, J. E., \& Cassinelli, J. P. 1993, ApJ, 409, 429

Bonneau, D., Clausse, J.-M., Delfosse, X., et al. 2006, A\&A, 456, 789

Carciofi, A. C. 2011, in IAU Symposium, Vol. 272, ed. C. Neiner, G. Wade, G. Meynet, \& G. Peters, 325 
Carciofi, A. C., \& Bjorkman, J. E. 2006, ApJ, 639, 1081

Carciofi, A. C., \& Bjorkman, J. E. 2008, ApJ, 684, 1374

Carciofi, A. C., Okazaki, A. T., Le Bouquin, J., et al. 2009, A\&A, 504, 915

Che, X., Monnier, J. D., Zhao, M., et al. 2011, ApJ, 732, 68

Chelli, A., Utrera, O. H., \& Duvert, G. 2009, A\&A, 502, 705

Delaa, O., Stee, Ph., Meilland, A., et al. 2011, A\&A, 529, A87

Demarque, P., Woo, J.-H., Kim, Y.-C., \& Yi, S. K. 2004, ApJS, 155, 667

Draper, Z. H., Wisniewski, J. P., Bjorkman, K. S., et al. 2011, ApJ, 728, L40

Duflot, M., Figon, P., \& Meyssonnier, N. 1995, A\&AS, 114, 269

Frémat, Y., Zorec, J., Hubert, A.-M., \& Floquet, M. 2005, A\&A, 440, 305

Ghosh, K., Iyengar, K. V. K., Ramsey, B. D., \& Austin, R. A. 1999, AJ, 118, 1061

Grundstrom, E. D. 2007, PhD thesis, Georgia State University

Hesselbach, E. N. 2009, PhD thesis, The University of Toledo

Isella, A., Testi, L., Natta, A., et al. 2007, A\&A, 469, 213

Iwamatsu, H., \& Hirata, R. 2008, PASJ, 60, 749

Jones, C. E., Tycner, C., \& Smith, A. D. 2011, AJ, 141, 150

Kato, S. 1983, PASJ, 35, 249

Kervella, P., \& Fouqué, P. 2008, A\&A, 491, 855

Kraus, S., Hofmann, K., Malbet, F., et al. 2009, A\&A, 508, 787

Le Bouquin, J., Bauvir, B., Haguenauer, P., et al. 2008, A\&A, 481, 553

Le Bouquin, J.-B., Absil, O., Benisty, M., et al. 2009, A\&A, 498, L41

Lee, U., Osaki, Y., \& Saio, H. 1991, MNRAS, 250, 432

McDavid, D. 1999, PASP, 111, 494

Meilland, A., Stee, P., Chesneau, O., \& Jones, C. 2009, A\&A, 505, 687

Meilland, A., Stee, P., Vannier, M., et al. 2007, A\&A, 464, 59

Meilland, A., Stee, P., Zorec, J., \& Kanaan, S. 2006, A\&A, 455, 953

Meilland, A., et al. 2011, A\&A, 532, A80

Miyoshi, M., Moran, J., Herrnstein, J., et al. 1995, Nature, 373, 127

Monnier, J. D., Berger, J.-P., Millan-Gabet, R., et al. 2006a, ApJ, 647, 444

Monnier, J. D., Zhao, M., Pedretti, E., et al. 2007, Science, 317, 342

Monnier, J. D., et al. 2006b, Proc. SPIE, 6268, 62681P

Okazaki, A. T. 1991, PASJ, 43, 75

Omidvar, K., \& McAllister, A. M. 1995, Phys. Rev. A, 51, 1063

Oudmaijer, R. D., Wheelwright, H. E., Carciofi, A. C., Bjorkman, J. E., \&

Bjorkman, K. S. 2011, in IAU Symposium, Vol. 272, ed. C. Neiner, G. Wade, G. Meynet, \& G. Peters, 418
Pavlovski, K., Harmanec, P., Bozic, H., et al. 1997, A\&AS, 125, 75

Pestalozzi, M. R., Elitzur, M., \& Conway, J. E. 2009, A\&A, 501, 999

Petrov, R. G., Malbet, F., Weigelt, G., et al. 2007, A\&A, 464, 1

Pollmann, E. 2002, Be Star Newsl. 35

Porter, J. M., \& Rivinius, T. 2003, PASP, 115, 1153

Pott, J., Woillez, J., Ragland, S., et al. 2010, ApJ, 721, 802

Quirrenbach, A., Bjorkman, K. S., Bjorkman, J. E., et al. 1997, ApJ, 479, 477

Quirrenbach, A., Buscher, D. F., Mozurkewich, D., Hummel, C. A., \& Armstrong, J. T. 1994, A\&A, 283, L13

Rivinius, T., Baade, D., Štefl, S., et al. 1998, A\&A, 333, 125

Rivinius, T., Štefl, S., \& Baade, D. 2006, A\&A, 459, 137

Rodríguez-Merino, L. H., Chavez, M., Bertone, E., \& Buzzoni, A. 2005, ApJ, 626,411

Saio, H., Cameron, C., Kuschnig, R., et al. 2007, ApJ, 654, 544

Schaefer, G. H., Gies, D. R., Monnier, J. D., et al. 2010, AJ, 140, 1838

Sofue, Y., \& Rubin, V. 2001, ARA\&A, 39, 137

Stee, P. 1996, A\&A, 311, 945

Stee, P., \& de Araujo, F. X. 1994, A\&A, 292, 221

Štefl, S., Rivinius, T., Baade, D., \& Carciofi, A. 2011, arXiv:1107.5297

Štefl, S., Rivinius, Th., Carciofi, A. C., et al. 2009, A\&A, 504, 929

Sturmann, J., Ten Brummelaar, T., Sturmann, L., \& McAlister, H. A. 2010, Proc. SPIE, 7734, 77343A

Tatulli, E., Millour, F., Chelli, A., et al. 2007, A\&A, 464, 29

ten Brummelaar, T. A., McAlister, H. A., Ridgway, S. T., et al. 2005, ApJ, 628, 453

Townsend, R. H. D., Owocki, S. P., \& Howarth, I. D. 2004, MNRAS, 350, 189

Tycner, C., Hajian, A. R., Armstrong, J. T., et al. 2004, AJ, 127, 1194

Tycner, C., Lester, J. B., Hajian, A. R., et al. 2005, ApJ, 624, 359

Vakili, F., Mourard, D., Stee, Ph., et al. 1998, A\&A, 335, 261

Wade, R. A., \& Rucinski, S. M. 1985, A\&AS, 60, 471

Waters, L. B. F. M. 1986, A\&A, 162, 121

Weigelt, G., Kraus, S., Driebe, T., et al. 2007, A\&A, 464, 87

Weintroub, J., Moran, J. M., Wilner, D. J., et al. 2008, ApJ, 677, 1140

Wilson, T. L., Rohlfs, K., \& Hüttemeister, S. 2009, Tools of Radio Astronomy (Berlin: Springer)

Yi, S. K., Kim, Y.-C., \& Demarque, P. 2003, ApJS, 144, 259

Yudin, R. V. 2001, A\&A, 368, 912 